\title{
Resonant Control of Structural Vibration Using Charge-Driven Piezoelectric Actuators
}

\author{
S. O. Reza Moheimani, Senior Member, IEEE, and Benjamin J. G. Vautier
}

\begin{abstract}
Driving piezoelectric actuators by charge, or current rather than voltage is known to significantly reduce the hysteretic nature of these actuators. Although this feature of piezoelectric transducers has been known to the researchers for some time, still voltage amplifiers are being used as the main driving mechanism for piezoelectric devices. This is due to the perceived difficulty in building charge/current amplifiers capable of driving highly capacitive loads such as piezoelectric actuators. Recently, a new charge amplifier has been proposed which is ideal for driving piezoelectric loads used in applications such as active damping of vibration. Consequently, it is now possible to effectively, and accurately control the charge deposited on the electrodes of a piezoelectric transducer, and thereby avoid hysteresis altogether. This paper further investigates properties of piezoelectric transducers driven by charge sources when used with resonant controllers for structural vibration control applications. The paper reports experimental results of a multivariable resonant controller implemented on a piezoelectric laminate cantilever beam.
\end{abstract}

Index Terms-Charge control, flexible structures, hysteresis, piezoelectric, resonant controllers, vibration control.

\section{INTRODUCTION}

A LARGE number of materials exhibit piezoelectricity, to some degree. When a piezoelectric material is mechanically stressed it develops an electrical charge across its terminals. This is known as the direct piezoelectric effect. Conversely, an electric field, or charge, applied to the same material will result in a change of its mechanical dimensions, which will result in mechanical strain. This property is referred to as the converse piezoelectric effect. Therefore, piezoelectric materials can be used as sensors, actuators, or in some applications as both.

There exist a certain class of ceramic materials, which, in their raw form, do not possess piezoelectric properties; however, piezoelectricity can be initiated in them. Ceramic materials, which fall within this category are: Lead-Zirconate-Titanate (PZT), Lead-Titanate $\left(\mathrm{PbTiO}_{2}\right)$, Lead-Zirconate $\left(\mathrm{PbZrO}_{3}\right)$, and Barium-Titanate $\left(\mathrm{BaTiO}_{3}\right)$. A true piezoelectric material is formed as a single crystal. A piezoelectric ceramic material, on the other hand, has a multicrystalline structure made up of large numbers of randomly oriented crystal grains, each with its own electric dipole. This random orientation of the grains results in the net cancellation of the electric dipoles. To initiate the piezoelectric effect in the ceramic material, its

Manuscript received October 14, 2004. Manuscript received in final form August 9, 2005. Recommended by Associate Editor C. Knospe. This work was supported by the Australian Research Council.

The authors are with the School of Electrical Engineering and Computer Science, University of Newcastle, Callaghan NSW 2308, Australia (e-mail: Reza.Moheimani@newcastle.edu.au; bvautier@ee.newcastle.edu.au).

Digital Object Identifier 10.1109/TCST.2005.857407 temperature is raised to just below the Curie temperature [1], and is then subjected to a large dc electric field. This is known as the "poling process." After the material is poled, the electric dipoles are aligned with the applied electric field, and the material will possess piezoelectric properties.

When driven by a voltage amplifier, piezoelectric actuators display nonlinear behavior known as hysteresis. The existence of hysteresis in piezoelectric materials is generally attributed to residual misalignment of crystal grains in the poled ceramic [1], [2]. It has been argued that hysteresis is an electrical property of piezoelectric materials, which mainly exists between the applied electric field and the resulting electrical charge [3]. Indeed, it has been demonstrated that by controlling electrical charge, or current rather than the applied voltage, the hysteresis effect can be substantially reduced [4].

The existence of hysteresis has been shown to have an adverse effect on the stability and closed-loop performance of voltage-controlled piezoelectric actuators. In [5], the authors report experimental results to illustrate that when a piezoelectric stack actuator is being used in a feedback loop with a voltage amplifier the gain and phase margins of the system deteriorate significantly compared to the situation in which the same actuator is driven by a charge amplifier. In particular, the authors report $28 \%$ improvement in the phase margin by using charge as the driving mechanism.

A large number of techniques have been developed that are aimed at reducing the effect of hysteresis associated with voltage-driven piezoelectric actuators (see [6] and the references therein). In particular, methods such as inversion-based Preisach modeling [7] and phase control [8] are two examples of the proposed techniques. The former is a complicated and time consuming procedure which assumes that the hysteresis associated with the piezoelectric actuators is of rate independent type, ${ }^{1}$ while the latter approach is only applicable if the driving signal is of fixed amplitude. There have also been efforts to model hysteresis as a disturbance, and thereby minimize its effect using a disturbance decoupling controller [10].

The best approach to deal with hysteresis in piezoelectric actuators appears to be to use charge, or current to drive the actuator since this would render the actuator linear. Even though this approach has been known for some time, it has not been widely used due to the perceived difficulty of driving highly capacitive loads. The main problem being the existence of offset voltages in the charge or current source circuit, which will eventually charge up the capacitive load. This will then distort the control signal being applied to the piezoelectric load. This issue [9].

${ }^{1}$ Hysteresis present in piezoelectric materials is known to be rate dependent 
has been pointed out by a number of authors [11], [12]. Recent research [13] proposes a new structure for charge and current sources capable of regulating the dc profile of the actuator. This concept will be discussed further in Section IV. Due to this development, it is now possible to use electrical charge as the driving control signal for piezoelectric actuators in structural control applications.

Flexible structures are highly resonant systems, which have an infinite number of vibratory modes. In most practical applications, however, one is often only interested in controlling a limited number of them. A controller can be designed using a model that describes dynamics of the system within the bandwidth of interest. Existence of out-of-bandwidth dynamics, however, could be problematic as high frequency modes may destabilize the closed-loop system, if their presence is not taken into account during the control design phase. One way of overcoming this hurdle is to use collocated piezoelectric sensors and actuators. The collocated structure allows for the design of controllers with guaranteed stability in presence of out-of-bandwidth dynamics. Resonant controllers have been designed for and implemented on voltage-driven piezoelectric laminated structures [14], [15]. If charge, or current sources are to be used to drive piezoelectric actuators, the structure of resonant controllers needs to be modified accordingly. This paper explains how this can be done.

A property of resonant controllers designed for charge-driven piezoelectric actuators is that they can be engineered to roll off at higher frequencies. This is in contrast to their voltage-driven counterparts which do not possess this important property. This is particularly significant in flexible structures where one would wish to reduce the effect of out-of-bandwidth dynamics on the stability of the closed-loop system.

The remainder of this paper continues as follows. Section II is concerned with the dynamics of multivariable piezoelectric laminates with collocated piezoelectric sensors and actuators. Section III is concerned with resonant control of multivariable piezoelectric laminates with voltage-driven piezoelectric actuators. Section IV discusses benefits of using charge, or current for driving piezoelectric actuators. Section V studies dynamics of charge-controlled piezoelectric actuators and Section VI suggests how the resonant control structure can be modified to work with charge-driven piezos. Section VII proposes a number of ways that parameters of a resonant controller can be determined, and Section VIII reports our experimental results. Finally, Section IX discusses some observations and Section X concludes this paper.

\section{Voltage-Driven Piezoelectric Actuators}

Piezoelectric actuators are often driven by voltage amplifiers, which are generally expensive due to the highly capacitive nature of piezoelectric loads, and the large voltages necessary to operate them. Moreover they only offer control over a limited bandwidth. Nevertheless, the bulk of the literature on feedback control of piezoelectric laminates is based on voltage-driven piezoelectric actuators, e.g., see [14], [16]-[23], and the references therein.

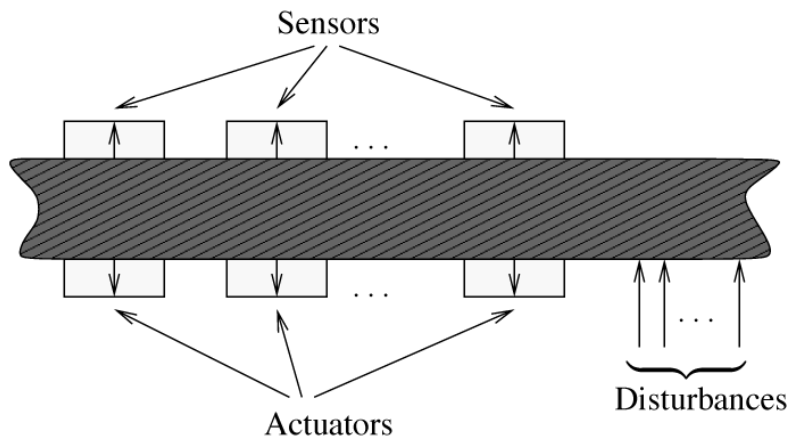

Fig. 1. Flexible structure with several collocated piezoelectric actuator/sensor pairs.

To allow for efficient control of several vibratory modes of a structure, it may be necessary to use a multivariable control strategy. Consider the system depicted in Fig. 1, where $m$ collocated piezoelectric sensor/actuator pairs are bonded to a flexible structure. It is well-known [14], [24]-[27] that the multivariable transfer function matrix of this system can be expressed as

$$
G_{v v}(s)=\sum_{i=1}^{M} \frac{\psi_{i} \psi_{i}^{\prime}}{s^{2}+2 \zeta_{i} \omega_{i} s+\omega_{i}^{2}}
$$

where $\psi_{i}$ is an $m \times 1$ vector, and $M \rightarrow \infty$. In practice, however, the integer $M$ is finite, but possibly a very large number which represents the number of modes that sufficiently describe the elastic properties of the structure under excitation [28], [29].

Also shown in Fig. 1 is a number of disturbances acting on the structure. This could represent point forces, distributed forces (such as a wind gust) or torques. The transfer function matrix relating the disturbance vector $W$ to the vector of voltages $V_{p}$ measured at the sensors can be written as

$$
G_{v w}=\sum_{i=1}^{M} \frac{\psi_{i} \gamma_{i}^{\prime}}{s^{2}+2 \zeta_{i} \omega_{i} s+\omega_{i}^{2}}
$$

where $\gamma_{i}$ is a $\ell \times 1$ matrix, assuming there are $\ell$ disturbances acting on the structure.

In a typical control design problem, structural models are often simplified by truncating the series (1) or (2). Doing this, however, may result in substantial error associated with system zeros, which may reduce the performance of a designed controller once implemented on the system. These truncation errors are particularly significant for collocated transfer functions $\left(G_{v v}\right)$. As explained in [30] and [31], these errors can be minimized by adding a feed-through term to the truncated model. That is, by approximating (1) by

$$
G_{v v}^{N}(s)=\sum_{i=1}^{N} \frac{\psi_{i} \psi_{i}^{\prime}}{s^{2}+2 \zeta_{i} \omega_{i} s+\omega_{i}^{2}}+D
$$

where $N \ll M$.

For the single-input-single-output (SISO) case, i.e., when $G_{v v}(s)$ represents the transfer function associated with only one collocated actuator/sensor pair, this system is known to possess interesting properties [32], one being the system is minimum phase, another being the poles and zeros of the system interlace. This ensures that the phase of the collocated transfer function will be within the 0 to $-180^{\circ}$ range. Subsequently, a differentiator would stabilize the system by increasing the 
effective damping of the system. This is referred to as direct velocity feedback control [33]. Obviously, there are complications associated with this technique, the most notable being the controller is improper, and therefore needs to be approximated by a proper one. Hence, the need for careful gain stabilization at higher frequencies. Furthermore, the control effort may be larger than necessary, particularly at frequencies far away from resonance frequencies.

To alleviate this difficulty a number of fixed structure controllers have been proposed in the literature, all of which have resonant structures reminiscent of the underlying system that is to be damped. Acceleration feedback [33] and positive position feedback [34] controllers being the two most notable examples.

\section{RESONANT CONTROLlERS FOR VOLTAGE-DRIVEN PIEZOELECTRIC ACTUATORS}

The structure of the collocated system, as expressed by (1) allows for the design of feedback controllers with specific structures that guarantee unconditional stability of the closed loop system. Such controllers are of interest due to their ability to avoid closed loop instabilities arising from the spill-over effect [35].

Typically, one may only be interested in controlling the first $N$ modes of (1). If a controller is designed based only on the $N$ mode model of the system, the existence of modes $N+1$ and higher may destabilize the closed loop system, once the controller is implemented. Resonant controllers have the distinctive property that closed loop stability of the system is guaranteed in presence of these out-of-bandwidth modes.

Two possible resonant controllers are

$$
K_{v}^{\alpha}=\sum_{i=1}^{\tilde{N}} \frac{\alpha_{i} \alpha_{i}^{\prime} s^{2}}{s^{2}+2 d_{i} \tilde{\omega}_{i} s+\tilde{\omega}_{i}^{2}}
$$

and

$$
K_{v}^{\beta}=\sum_{i=1}^{\tilde{N}} \frac{\beta_{i} \beta_{i}^{\prime} s\left(s+2 d_{i} \tilde{\omega}_{i}\right)}{s^{2}+2 d_{i} \tilde{\omega}_{i} s+\tilde{\omega}_{i}^{2}}
$$

where typically $\tilde{N} \ll M$. Note that both $\alpha_{i}$ and $\beta_{i}$ are $m \times 1$ vectors.

The typical feedback control problem associated with system (1) and a resonant controller $K_{v}$ is illustrated in Fig. 2. Here, $V_{p}$ is the vector of voltages measured at the piezoelectric sensors, while $W$ is the vector of disturbances acting on the structure. The purpose of the controller is to negate the effects of disturbances by increasing the effective damping of the structure. This is achieved by shifting the closed loop poles of the system deeper into the left half of the complex plane.

Closed-loop stability for the multivariable collocated system (1) under (4) and (5) can be proved in a number of ways. A proof is given in the Appendix.

To this end we point out that resonant controllers (4) and (5) are multivariable versions of the SISO resonant controller proposed by the first author and colleagues in [15]. They are also closely related to resonant shunt controllers as described in [36] and [37]. These controllers, if designed properly, are known to offer good performance in terms of adding damping to the structure.

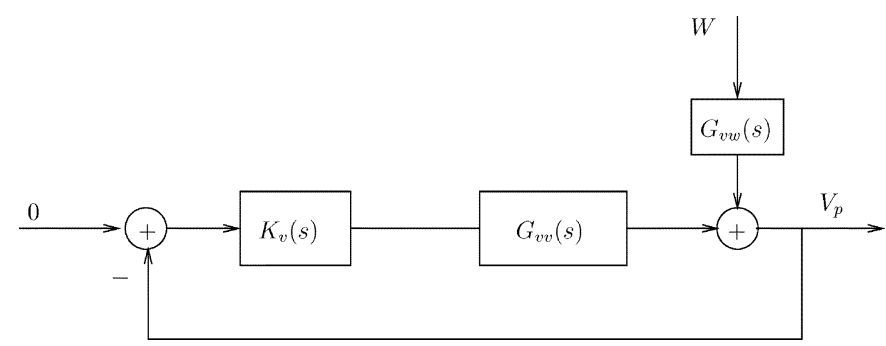

Fig. 2. Feedback control of vibration using voltage-driven collocated piezoelectric actuator/sensor pairs.
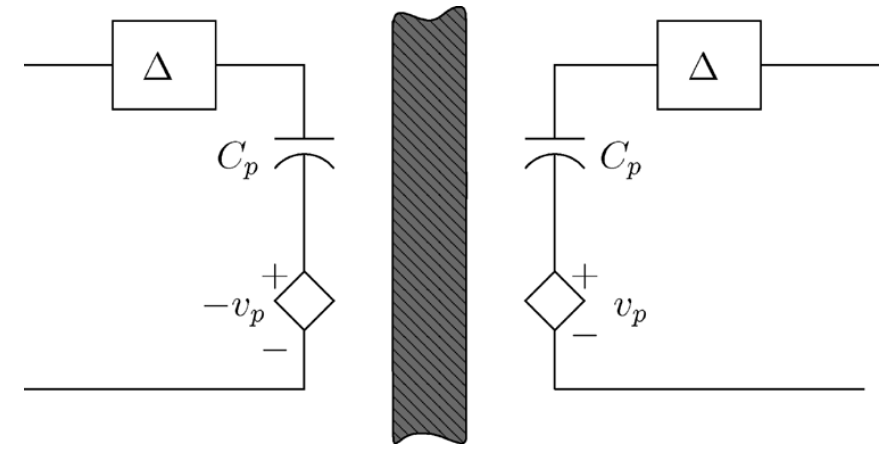

Fig. 3. Electrical equivalent of a collocated piezoelectric actuator/sensor pair incorporating the effect of hysteresis.

\section{Charge Control Versus Voltage Control}

The resonant controllers (4) and (5) will raise two significant problems when voltage is used to drive the piezoelectric actuators.

Typically the frequency response of a flexible structure with collocated piezoelectric transducers rolls off very slowly at high frequencies. ${ }^{2}$ A controller that rolls off is therefore preferable. The first problem therefore arises form the fact that voltage driven resonant controllers do not roll off quickly at higher frequencies. Although, the stability of the closed loop system under (4) and (5) is guaranteed, because of the asymptotic behavior of (4) and (5) the performance of the closed loop system may suffer from high-frequency noise and the inevitable phase lag introduced by the sensor.

The second complication is due to the hysteretic nature of piezoelectric actuators when driven by voltage amplifiers. As illustrated in Fig. 3, each piezoelectric transducer can be modeled as the series connection of a voltage source, which is proportional to the total strain in the piezo, a capacitor and a nonlinear element $\Delta$ [3]. The nonlinearity is of the hysteresis type and is much more profound when the actuator is operated at high voltages. ${ }^{3}$

This clearly suggests that when possible electrical charge rather than voltage should be used to drive piezoelectric actuators. The reason why charge, or current sources have not been extensively used to drive piezoelectric transducers can be

\footnotetext{
${ }^{2}$ This situation can be improved by reducing the dimensions of piezoelectric transducers [38]. This would come at the price of reduced controller authority over the structure, and cannot be recommended for all applications.

${ }^{3}$ It is usually argued that when a piezoelectric actuator is being operated at low voltages, hysteresis becomes almost negligible. Restricting the level of control voltage, however, is not advisable since the actuator is not being used to its full potential.
} 
attributed to the perceived difficulty in implementing devices capable of driving highly capacitive loads such as piezoelectric actuators. Existence of offsets in conjunction with the uncontrolled nature of the output voltage generally results in the capacitor being charged up. Once the output voltage reaches the power supply rail, the output becomes saturated, and the amplifier fails to perform properly.

This issue can be resolved by adding an extra feedback loop to the standard charge/current amplifier structure, as proposed in [13]. To understand the operation of the system, consider the simplified schematic of a compliance feedback for a current or charge source shown in Fig. 4. The device functions as a charge amplifier if the impedance $Z_{s}(s)$ is a capacitor, $C_{s}$ and as a current amplifier if $Z_{s}(s)$ is a resistor, $R_{s}$. When operated in the charge mode, and neglecting the compliance controller $C(s)$, the high gain feedback $K$ works to equate the applied reference $q_{\text {ref }}=v_{\text {ref }}$ to the voltage measured across the sensing capacitor $C_{s}$. In the Laplace domain, at frequencies well within the bandwidth of the control loop, the load current $i_{L}(s)$ is equal to $q_{\mathrm{ref}}(s) / Z_{s}(s)$. When $Z_{s}(s)$ is a resistor $R_{s}$, $i_{L}(s)=v_{\text {ref }}(s) / R_{s}$ and the circuit operates as a current amplifier with gain $1 / R_{s} A / V$. If $Z_{s}(s)$ is a capacitor $C_{s}$, then $q_{L}=$ $v_{\text {ref }}(s) C_{s}$, which means the circuit functions as a charge amplifier with gain $C_{s}$ Coulumb/V. Effective dc regulation of the compliance voltage can be achieved using a PI compliance controller. Indeed, it can be proved that a PI controller completely suppresses the dc component of $v_{o}$. For a thorough discussion of properties of the compliance feedback-based charge/current amplifier the reader is referred to [13].

It needs to be pointed out that although this charge amplifier is well suited for damping purposes, it is of little use in applications that include positioning, e.g., [39], [40]. A DC accurate charge amplifier is currently under development, and will be reported in due course.

\section{Feedback Structure of Charge-Driven PIEZOELECTRIC LAMINATES}

In order to analyze the effect of using charge rather than voltage in vibration control applications, let us define the following parameters:

$$
\begin{gathered}
V=\left[\begin{array}{c}
v_{1} \\
v_{2} \\
\vdots \\
v_{m}
\end{array}\right] \quad V_{p}=\left[\begin{array}{c}
v_{p_{1}} \\
v_{p_{2}} \\
\vdots \\
v_{p_{m}}
\end{array}\right] \quad W=\left[\begin{array}{c}
w_{1} \\
w_{2} \\
\vdots \\
w_{\ell}
\end{array}\right] ; \\
\Lambda=\left[\begin{array}{cccc}
\frac{1}{C_{p_{1}}} & \frac{1}{} & \\
& \frac{C_{p_{1}}}{} & \\
& & \ddots & \\
& & \frac{1}{C_{p_{m}}}
\end{array}\right] \quad Q=\left[\begin{array}{c}
q_{1} \\
q_{2} \\
\vdots \\
q_{m}
\end{array}\right]
\end{gathered}
$$

where $V$ is the vector of voltages applied to the piezoelectric actuators, $V_{p}$ is the vector of voltages measured from the piezoelectric sensors, $W$ is the vector of disturbances acting on the beam, $C_{p_{i}}$ is the capacitance associated with each collocated piezoelectric patch, and $Q$ represents the vector of electrical charges.

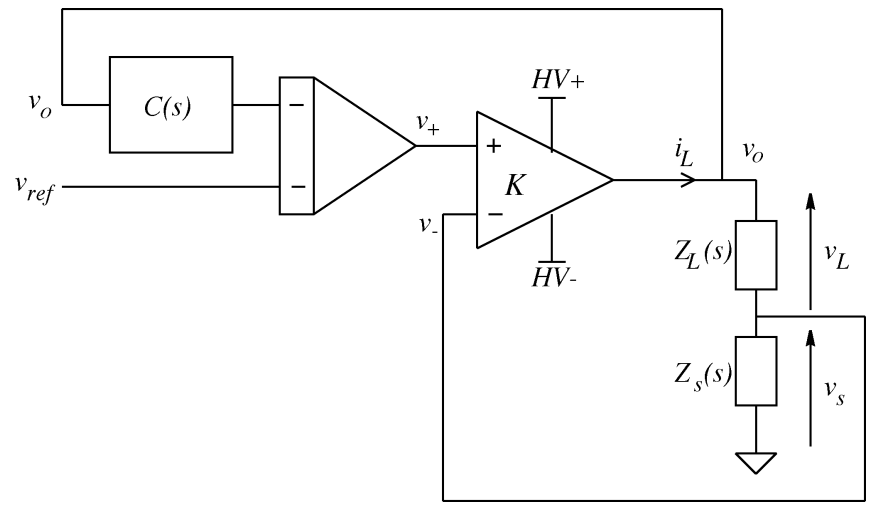

Fig. 4. Current/charge amplifier with added compliance feedback.
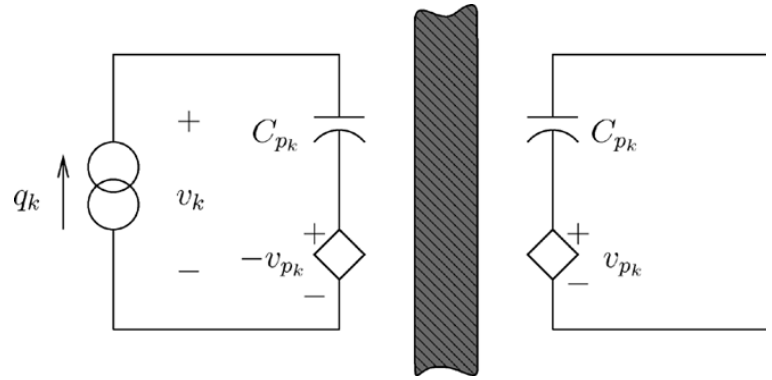

Fig. 5. Collocated charge control of one piezoelectric actuator/sensor pair.

Some of the assumptions made here are: i) $m$ piezoelectric actuator/sensor pairs are bonded to the structure; ii) each pair consists of two identical transducers, however, not all transducers are necessarily identical; iii) $\ell$ disturbances are acting on the structure; iv) the controller is fully multivariable; and v) $C_{p_{i}}$ represents capacitance of the $i$ th transducer.

Writing the KVL around the $k$ th loop, as illustrated in Fig. 5, we obtain ${ }^{4}$

$$
v_{k}(s)=-v_{p_{k}}(s)+\frac{1}{C_{p_{k}}} q_{k}(s) .
$$

Using the previous notation, this implies

$$
V(s)=-V_{p}(s)+\Lambda Q(s) .
$$

Since

$$
Q(s)=-K_{q}(s) V_{p}(s)
$$

where $K_{q}$ is the charge controller, and

$$
V_{p}(s)=G_{v w}(s) W(s)+G_{v v}(s) V(s)
$$

which follows from the linearity of the system, the feedback structure illustrated in Fig. 6 can be obtained. Here, $G_{v v}$ is the multivariable transfer function matrix of the collocated system (1). $G_{v w}$ is also a multivariable $m \times \ell$ transfer function matrix, with a structure specified in (2).

It can be verified that the multivariable transfer function matrix relating $V_{p}$ to $W$ is given by

$$
V_{p}(s)=\left[I+G_{v v}(s)\left(I+\Lambda K_{q}(s)\right)\right]^{-1} G_{v w}(s) W(s) .
$$

\footnotetext{
${ }^{4}$ Note that this is consistent with the sensor equation in [21].
} 


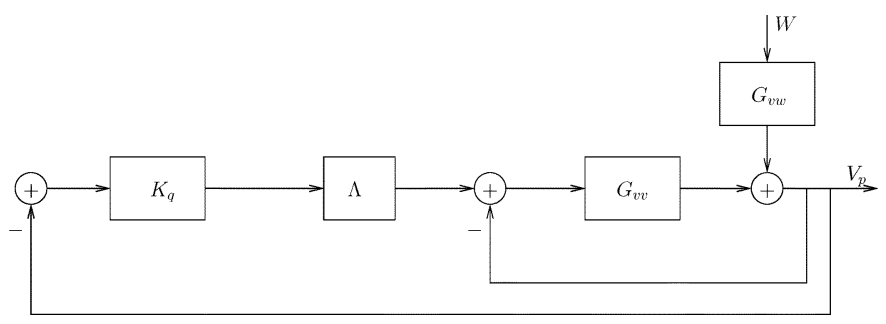

Fig. 6. Feedback structure associated with charge-driven piezoelectric actuator/sensor pairs.

A point that is needed to be clarified here is what happens when $K_{q}=0$ in (9). When the piezoelectric actuators are driven by voltage amplifiers, and $K_{v}$ (see Fig. 2) is set to zero, the closed loop transfer function matrix of the system reduces to $G_{v w}$. However, if $K_{q}$ in (9) is set to zero, the closed loop system reduces to

$$
\left[I+G_{v v}(s)\right]^{-1} G_{v w}(s) .
$$

When $K_{v}=0$, the piezoelectric actuators are effectively short-circuited. However, $K_{q}=0$ means the actuators are left open-circuited. Although the difference between the response in these two cases may come as a surprise, it does make a difference if piezoelectric transducers are open- or short-circuited. To appreciate this, we point out that (9) can be rewritten as

$$
\begin{aligned}
V_{p}(s)=[I+\Lambda(I+ & \left.\left.G_{v v}(s)\right)^{-1} G_{v v}(s) K_{q}(s)\right]^{-1} \\
& \times\left(I+G_{v v}(s)\right)^{-1} G_{v w}(s) W(s)
\end{aligned}
$$

which suggests the feedback structure in Fig. 7.

\section{RESONANT CONTROLLERS FOR CHARGE-DRIVEN PIEZOELECTRIC ACTUATORS}

Identification of the feedback structure associated with a charge-driven piezoelectric laminate leads to an important observation: If the piezoelectric actuators were to be driven by voltage amplifiers, rather than charge amplifiers, and if the underlying system were linear, then the two closed-loop systems would be identical as long as

$$
K_{v}(s)=I+\Lambda K_{q}(s) .
$$

This observation becomes especially useful in terms of developing resonant control structures for charge-driven systems. Given that for an arbitrary $K_{v}(s)$ the equivalent charge controller is

$$
K_{q}(s)=\Lambda^{-1}\left(K_{v}(s)-I\right)
$$

if $K_{v}(s)$ is chosen to be either $K_{v}^{\alpha}(s)$ or $K_{v}^{\beta}(s)$, as defined in (4) and (5), with the additional constraints

$$
\sum_{i=1}^{\tilde{N}} \alpha_{i} \alpha_{i}^{\prime}=I
$$

and

$$
\sum_{i=1}^{\tilde{N}} \beta_{i} \beta_{i}^{\prime}=I
$$

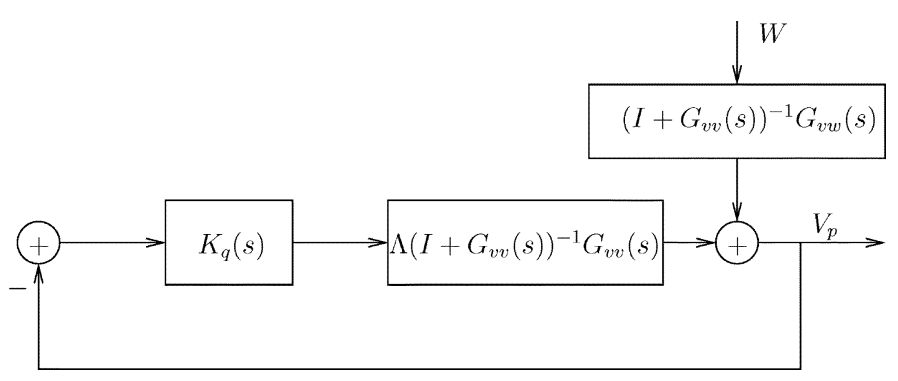

Fig. 7. Feedback control system with charge-driven piezoelectric actuators.

we obtain the following resonant controllers for equivalent charge-driven systems:

$$
K_{q}^{\alpha}(s)=-\Lambda^{-1} \sum_{i=1}^{\tilde{N}} \frac{\alpha_{i} \alpha_{i}^{\prime}\left(s+2 d_{i} \tilde{\omega}_{i}\right)}{s^{2}+2 d_{i} \tilde{\omega}_{i} s+\tilde{\omega}_{i}^{2}}
$$

and

$$
K_{q}^{\beta}(s)=-\Lambda^{-1} \sum_{i=1}^{\tilde{N}} \frac{\beta_{i} \beta_{i}^{\prime} \tilde{\omega}_{i}^{2}}{s^{2}+2 d_{i} \tilde{\omega}_{i} s+\tilde{\omega}_{i}^{2}} .
$$

It can be observed, from (16) and (17), that resonant controllers for charge-driven piezoelectric actuators will be strictly proper as long as conditions (14) and (15) are enforced. In the SISO case, controllers (16) and (17) will roll off quickly at 20 and $40 \mathrm{~dB}$ per decade respectively. This is a favorable property that is not present for the voltage driven case as discussed previously.

It should be noticed that conditions (14) and (15) could limit the closed loop performance of resonant controllers since they impose a hard constraint on the structure of the controller. Despite this, good performance can still be obtained using these controllers as illustrated, experimentally, in Section VII.

Closed-loop stability of the charge-driven system under (16) and (17) is closely related to the voltage-driven system, and is discussed in the Appendix.

\section{EXPERIMENTAL TESTBED}

Experiments were performed on a cantilever beam with two collocated piezoelectric pairs. One pair was located close to the clamped end and the other closer to the free end of the beam. For each collocated pair, one piezoelectric patch was used as an actuator, and was driven by a charge amplifier, while the voltage induced in the other patch was used as the measurement. Another piezoelectric actuator was bonded to the beam, somewhere between the two actuating patches. This transducer was driven by a voltage source to apply a disturbance to the beam. The transducer collocated with this actuator was short circuited so that it would not add any loading on the structure. A Schematic of the experimental testbed is demonstrated in Fig. 8, a picture of the actual beam is shown in Fig. 9.

The purpose of the experiment was to design and implement a two-input-two-output resonant controller to regulate the tip displacement of the beam in face of vibrations arising from a disturbance voltage applied to the third actuator. For the purpose of designing such a controller an accurate model of the cantilever beam was needed. The modeling process is detailed in the remainder of this section. 


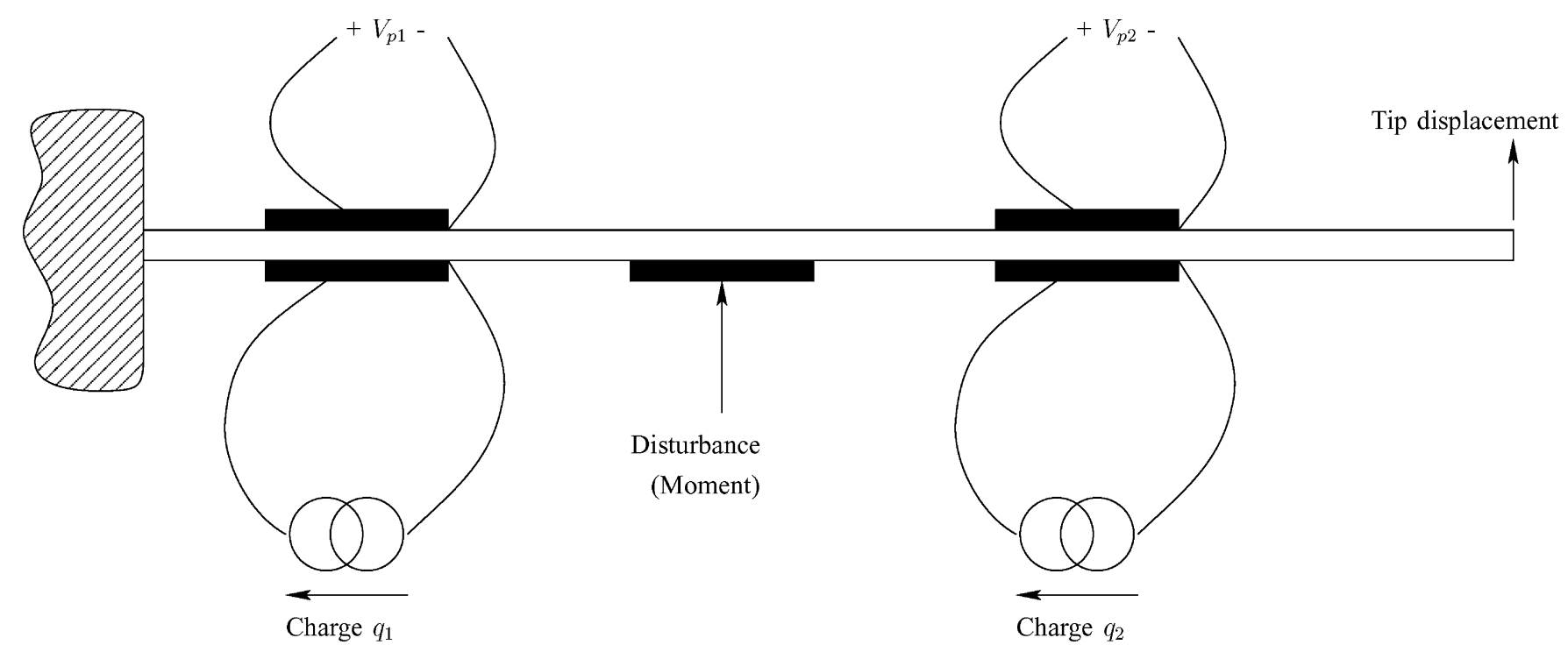

Fig. 8. Beam arrangement.

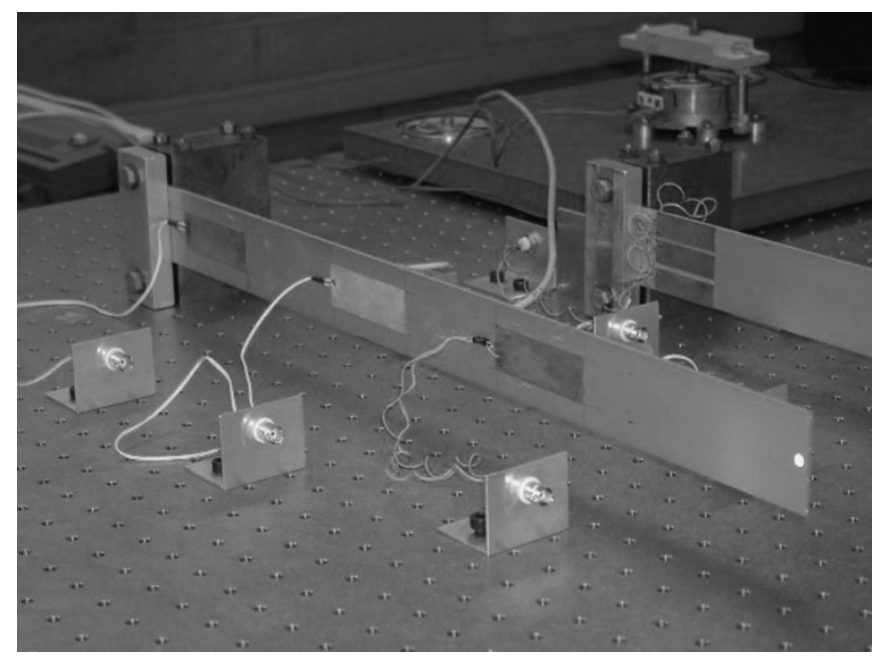

Fig. 9. Picture of the cantilever beam.

\section{A. The Hysteresis Effect}

To demonstrate the presence of hysteresis one of the piezoelectric actuators was first driven by a voltage source and then subsequently by a charge source. The voltage induced in the collocated piezoelectric transducer was measured and recorded. The actuating signal in each case was a linearly decaying singletone sinusoid of $24 \mathrm{~Hz}$. At this frequency the corresponding transfer function has zero phase. Therefore, any deviation from a straight line on the input-output plot is purely due to hysteresis.

In this experiment, the amplitude of the charge signal was adjusted to ensure that the measured voltage at the collocated piezoelectric transducer was at a comparable level to that measured when the actuator was driven by a voltage source. The results are illustrated in Fig. 10(a) and (b). The presence of hysteresis when the actuator is driven by a voltage source is evident from Fig. 10(a). However, when a charge source is used, hardly any hysteresis can be observed. This agrees with similar results discussed in [11] and [12]. In particular, it has been reported that piezoelectric actuators exhibit up to $80 \%$ less hysteresis when driven by charge amplifiers [4], [41]. In other words, in a piezoelectric transducer hysteresis mainly exists between the voltage and mechanical strain, rather than the charge (or current) and strain [3].

It should be pointed out that hysteresis is not a major source of difficulty with piezoelectric sensors. The use of a buffer circuit with very high input impedance will significantly reduce the effect of hysteresis in the sensor. A similar strategy for the actuator does not exist.

\section{B. State-Space Model of the Composite System}

Assuming, for a moment, that all actuators are being driven by voltage amplifiers, the multivariable plant can be represented by the following state-space equations:

$$
\begin{aligned}
\dot{x}(t) & =A x(t)+B_{w} w(t)+B_{v} V(t) \\
Y_{\text {tip }} & =C_{y} x(t)+D_{y w} w(t)+D_{y v} V(t) \\
V_{p}(t) & =C_{v} x(t)+D_{v w} w(t)+D_{v v} V(t)
\end{aligned}
$$

where $x \in \mathbf{R}^{2 N}$ represents the state vector of the system, $N$ is the number of modes included in the model, and $w$ and $V=$ $\left[\begin{array}{ll}v_{1} & v_{2}\end{array}\right]$ represent the disturbance and control input voltages, respectively.

Substituting (6) into (20), we obtain

$$
\begin{aligned}
V(t)=-\left(I+D_{v v}\right)^{-1} C_{v} x(t)- & \left(I+D_{v v}\right)^{-1} D_{v w} w(t) \\
& +\left(I+D_{v v}\right)^{-1} \Lambda Q(t) .
\end{aligned}
$$

Equation (21) can now be substituted into (18)-(20) to obtain the multivariable state space representation for the plant when the actuating patches are being driven by charge sources. The resulting system is

$$
\begin{aligned}
\dot{x}(t) & =\tilde{A} x(t)+\tilde{B}_{w} w(t)+\tilde{B}_{q} Q(t) \\
Y_{\mathrm{tip}}(t) & =\tilde{C}_{y} x(t)+\tilde{D}_{y w} w(t)+\tilde{D}_{y q} Q(t) \\
V_{p}(t) & =\tilde{C}_{v} x(t)+\tilde{D}_{v w} w(t)+\tilde{D}_{v q} Q(t)
\end{aligned}
$$




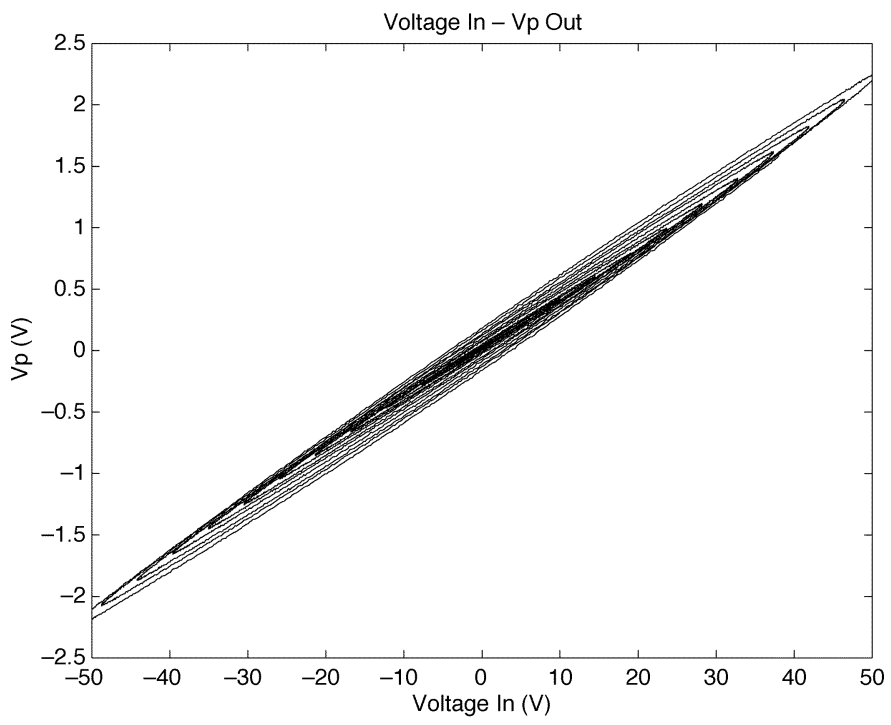

(a)



(b)

Fig. 10. Plot of the output voltage versus (a) the input voltage and (b) the input charge.

where

$$
\begin{aligned}
\tilde{A} & =A-B_{v}\left(I+D_{v v}\right)^{-1} C_{v} \\
\tilde{B}_{w} & =B_{w}-B_{v}\left(I+D_{v v}\right)^{-1} D_{v w} \\
\tilde{B}_{q} & =B_{v}\left(I+D_{v v}\right)^{-1} \Lambda \\
\tilde{C}_{y} & =C_{y}-D_{y v}\left(I+D_{v v}\right)^{-1} C_{v} \\
\tilde{D}_{y w} & =D_{y w}-D_{y v}\left(I+D_{v v}\right)^{-1} D_{v w} \\
\tilde{D}_{y q} & =D_{y v}\left(I+D_{v v}\right)^{-1} \Lambda \\
\tilde{C}_{v} & =C_{v}-D_{v v}\left(I+D_{v v}\right)^{-1} C_{v} \\
\tilde{D}_{v w} & =D_{v w}-D_{v v}\left(I+D_{v v}\right)^{-1} D_{v w} \\
\tilde{D}_{v q} & =D_{v v}\left(I+D_{v v}\right)^{-1} \Lambda .
\end{aligned}
$$

The reader would notice that the dynamics of the plant are different depending on whether charge or voltage is used to drive the actuating patches. This is reflected in the different state matrices associated with the systems represented by (18) and (22).

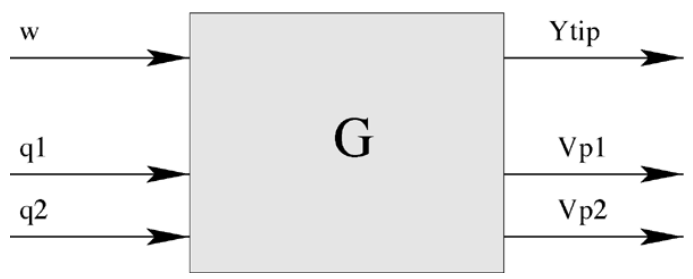

Fig. 11. Augmented multiple-input-multiple-output (MIMO) plant.

Furthermore, as evidenced from (18) and (22) the charge driven state-space equations are expressed in terms of $A, B, C$, and $D$ matrices associated with the voltage-driven case. Therefore, to successfully construct the charge-driven equations, it is necessary to accurately identify the values of these matrices.

These values can be derived from the structural properties and dimensions of the beam and its boundary conditions as demonstrated in [14]. In this paper we used a system identification approach, which is detailed in Section VII-D.

\section{Structure of the State-Space Model}

The physical modeling for the voltage driven scenario is well understood [14], [24] and uses derivations from the Euler-Bernoulli beam equation to determine the transfer function from input voltages $(V)$ to output voltages $\left(V_{p}\right)$ (or output tip displacement $Y_{\text {tip }}$ ). From these derivations, it has been shown that the $A, B$ and $C$ matrices (for the voltage driven case) are of the form

$$
\begin{aligned}
& A=\left[\begin{array}{ccccc}
0 & 1 & & 0 & 0 \\
-\omega_{1}^{2} & -2 \zeta_{1} \omega_{1} & & 0 & 0 \\
& & \ddots & & \\
0 & 0 & & 0 & 1 \\
0 & 0 & & -\omega_{N}^{2} & -2 \zeta_{N} \omega_{N}
\end{array}\right] \\
& B=\left[\begin{array}{lll}
B_{w} & B_{v_{1}} & B_{v_{2}}
\end{array}\right]=\left[\begin{array}{ccc}
0 & 0 & 0 \\
g_{1}^{w} & g_{1}^{v_{1}} & g_{1}^{v_{2}} \\
\vdots & \vdots & \vdots \\
0 & 0 & 0 \\
g_{N}^{w} & g_{N}^{v_{1}} & g_{N}^{v_{2}}
\end{array}\right] \\
& C=\left[\begin{array}{c}
C_{y} \\
C_{v_{1}} \\
C v_{2}
\end{array}\right]=\left[\begin{array}{ccccc}
f_{1}^{y} & 0 & \ldots & f_{N}^{y} & 0 \\
f_{1}^{v_{1}} & 0 & \ldots & f_{N}^{v_{1}} & 0 \\
f_{1}^{v_{2}} & 0 & \ldots & f_{N}^{v_{2}} & 0
\end{array}\right]
\end{aligned}
$$

where $\omega_{i}$ and $\zeta_{i}$ correspond to the natural frequency and damping ratio of the $i$ th vibrational mode of the structure, $g_{i}^{w}$, $g_{i}^{v_{1}}$ and $g_{i}^{v_{2}}$ are values that correspond to the input disturbance $(w)$ and control input voltages $\left(v_{1}\right.$ and $\left.v_{2}\right)$ respectively, and $f_{i}^{y}, f_{i}^{v_{1}}$ and $f_{i}^{v_{2}}$ are values that correspond to the output tip displacement and induced output voltages $\left(v_{p_{1}}\right.$ and $\left.v_{p_{2}}\right)$ respectively. Note that (26) and (27) can be equally expressed in terms of the notation used in (1) and (2).

Since the cantilever beam has two collocated pairs of piezoelectric transducers (Fig. 8) we can make the following simplification and set $g_{i}^{v_{1}}=f_{i}^{v_{1}}$ and $g_{i}^{v_{2}}=f_{i}^{v_{2}}$ [24], [38].

The matrices ( $A, B$ and $C$ ) are then used in (22)-(24) to obtain the charge driven state space equations for the plant.

In the experiment only the first three vibrational modes of the beam were considered, i.e., $N=3$. We therefore had to include a feed-through term $D$ to compensate for the misalignment of 


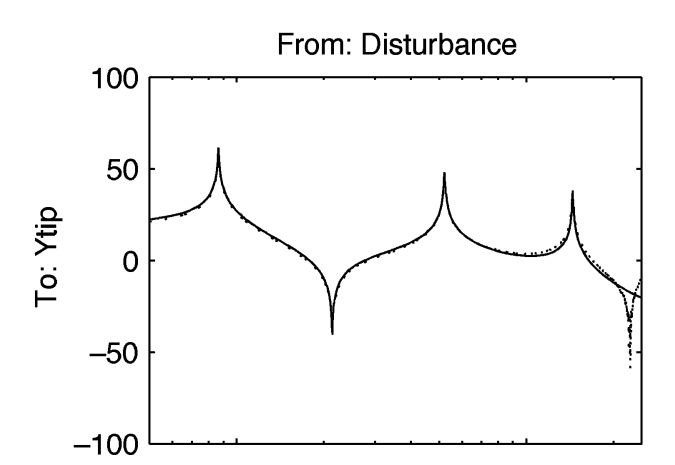

Bode Magnitude Diagram
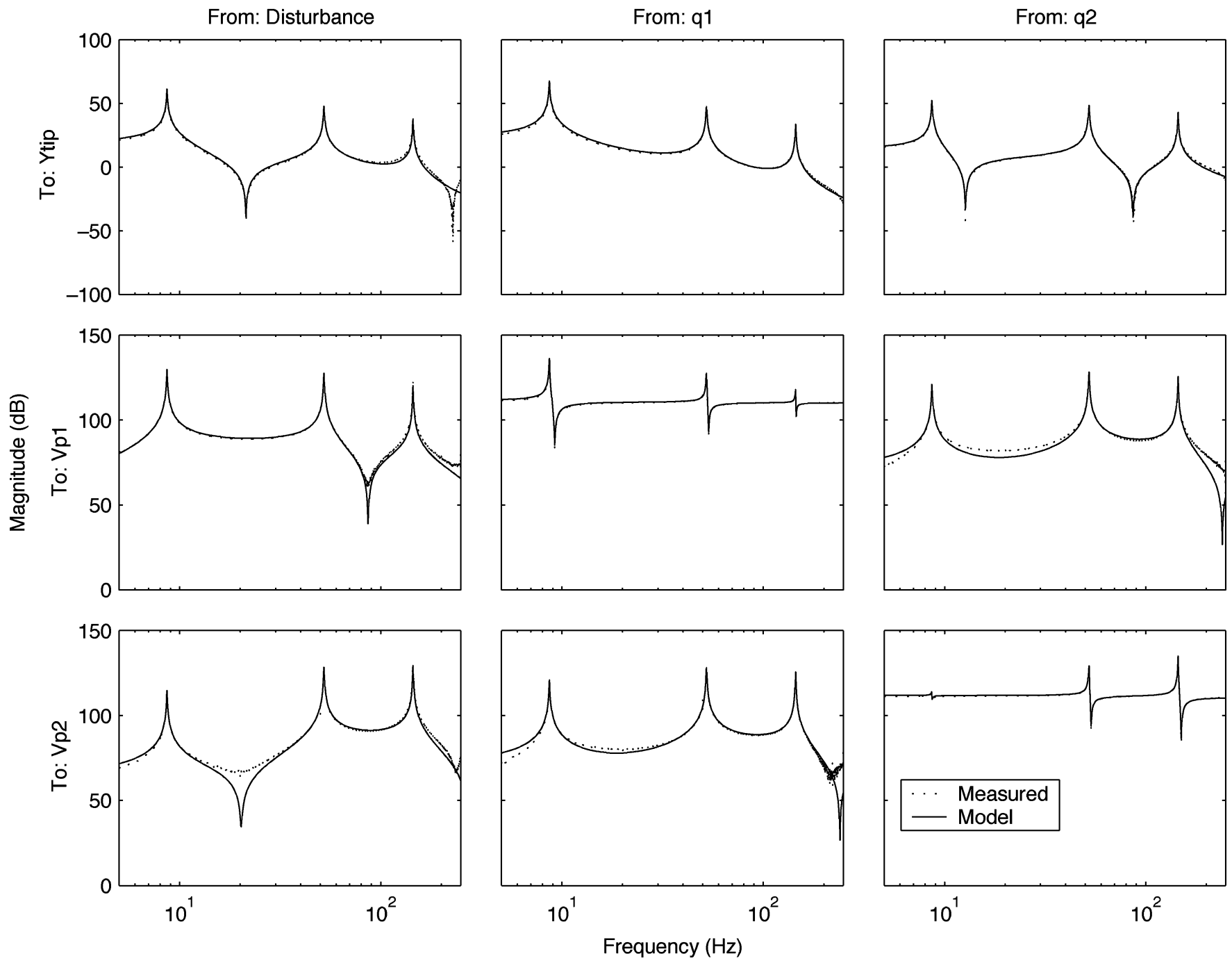

Fig. 12. Identified model with measured data.

the in-bandwidth zero locations that arises from the inability to model the higher order modes of the structure [30], [42].

\section{System Identification}

To obtain a model of the plant, suitable for control design purposes, a three-input-three-output model as illustrated in Fig. 11 was identified. The first input corresponds to the disturbance voltage $(w)$ applied to the middle patch. The second and third inputs are the charges $\left(q_{1}\right.$ and $\left.q_{2}\right)$ applied to the first and second actuators respectively. The first output corresponds to the displacement measured at the tip of the cantilever $\left(Y_{\text {tip }}\right)$. The second and third outputs are the voltages $\left(V_{p_{1}}\right.$ and $\left.V_{p_{2}}\right)$ measured at the first and second piezoelectric transducers, respectively.

To model the plant we measured all nine frequency responses for each input-output combination. These frequency responses were obtained by applying a sinusoidal chirp signal of varying frequency (from 5 to $250 \mathrm{~Hz}$ ) to the piezoelectric actuators and measuring the corresponding output signals of interest (namely the output voltages $V_{p}$ from the collocated sensors and the displacement at the tip of the beam $Y_{\text {tip }}$ ). The input/output data was processed in real time by the Polytec laser scanning vibrometer (PSV-300) software to obtain the desired frequency responses. An optimization problem was set up and solved to obtain the "best fit" state space model by minimizing the normalized least squared error between the simulated and measured data.

Prior to running the optimization, the values of $\omega$ and $\zeta$ for each mode were fixed (i.e., the $A$ matrix is fixed). This significantly reduces the numerical complexity of the optimization problem that is to be solved. In fact, the natural frequencies $\omega_{i}$ and damping ratios $\zeta_{i}$ can be obtained, and fine tuned with relative ease by measuring the location and height of the peaks from the measured frequency responses. Another approach is to perform a sub-space system identification on one of the channels, which would yield a good initial guess for the values of $\omega$ and $\zeta$ for each mode. Once the $A$ matrix is fixed, only numerical values of the $B, C$, and $D$ matrices need to be determined using the optimization.

The results of the magnitude Bode plots are illustrated in Fig. 12, and demonstrate that the identified model closely matches the experimentally measured data. Some interesting observations can be drawn from these plots. The Bode plots 


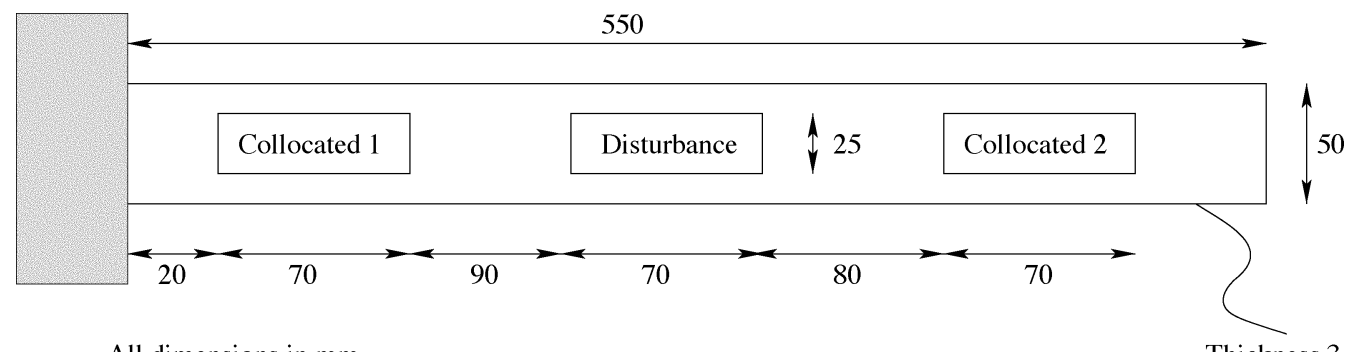

All dimensions in $\mathrm{mm}$

Thickness 3

The thickness of each collocated patch is $0.25 \mathrm{~mm}$ and the "disturbance" patch is $0.5 \mathrm{~mm}$

Fig. 13. Bean setup and dimension.

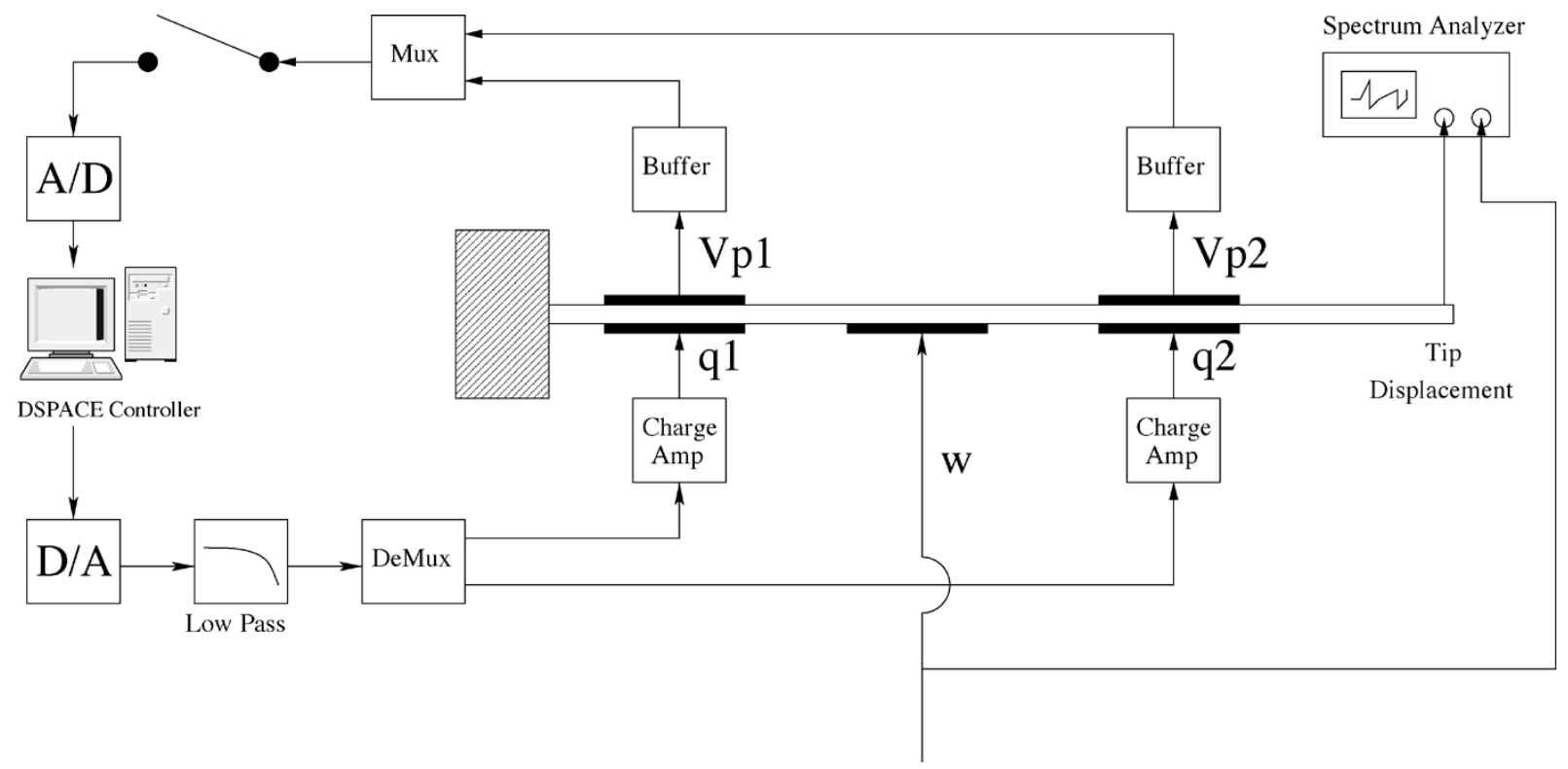

Fig. 14. Experimental schematic.

corresponding to the first and second collocated actuator/sensor pairs are found in the [2,2] and [3,3] positions, respectively. A closer look at these plots reveals that the first mode is clearly the dominant mode in the first collocated pair $([2,2])$. Therefore, one would expect the controller to have significant authority over the first vibratory mode of the structure through this actuator/sensor pair. However, the controller would have little authority over the second and third modes as evident from the low profile of these two modes in the ([2,2]) plot, if this pair was to be used in a SISO setting. This situation is reversed for the second collocated pair $([3,3])$. The reason for this can be explained by examining the first three vibrational mode shapes of a cantilever beam in Fig. 15 [24]. For an actuator to have maximum authority over a given mode it must be placed at the location where the curvature ${ }^{5}$ is greatest for that particular vibrational mode [38].

The aforementioned discussion should make it clear that it may be nearly impossible to control a large number of vibration modes with only one actuator/sensor pair. To effectively control a number of modes, one may need to use several collocated actuator/sensor pairs along with a multivariable controller. As indicated before, although the controller has little au-

${ }^{5}$ For a one-dimensional structure such as a beam strain is proportional to the curvature [26].

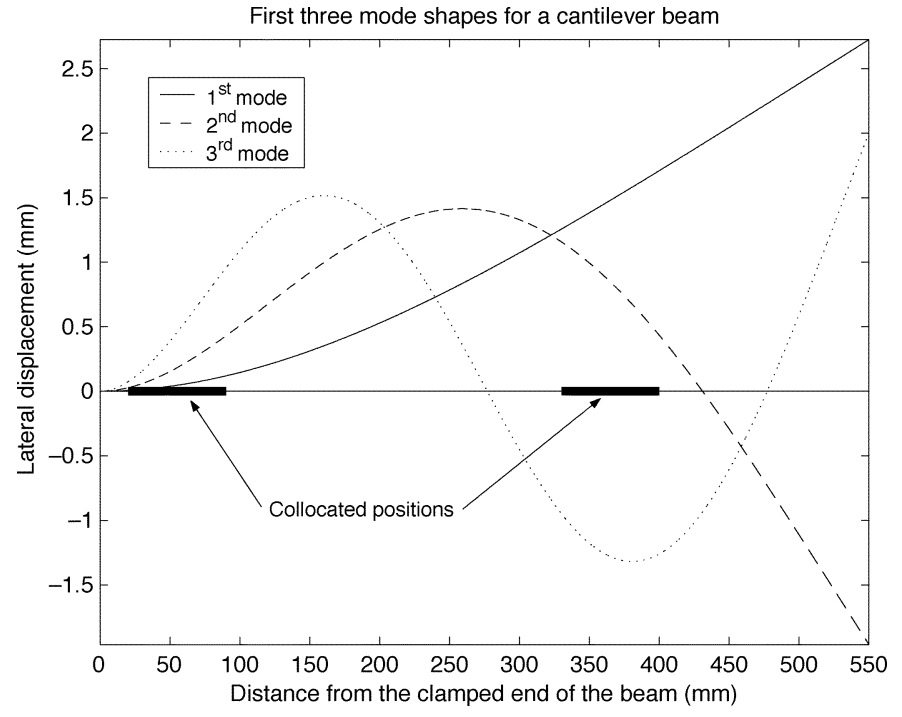

Fig. 15. First three mode shapes for a cantilever beam.

thority over the third mode through the first actuator/sensor pair, it would have good authority over this mode through the second actuator/sensor pair and also through the first actuator and the second sensor, as evident from ([2,3]). 


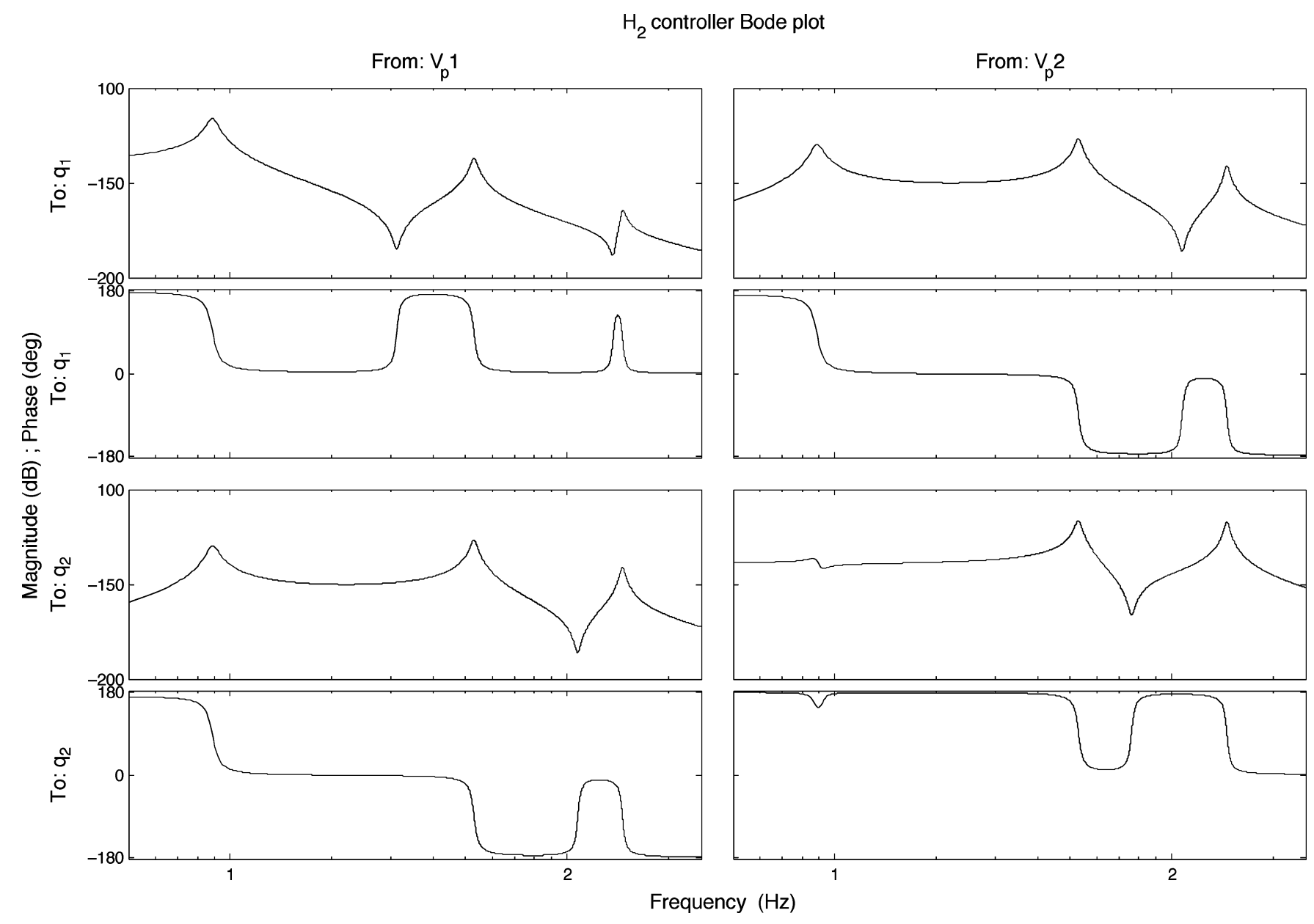

Fig. 16. Bode plot of the resonant $\mathrm{H}_{2}$ controller.

If we now return to the plots [2,2] and [3,3], the reader will notice that the collocated arrangement for these two plots can easily be deduced from there peak-trough alternating pattern. Further evidence to such a collocated arrangement is demonstrated because the plots [2,3], [3,2] are nearly identical.

The largest mismatch is found in the $[3,1]$ Bode magnitude plot, where the model fails to properly match the measured data around the first antinode. The reason for this is still not clear but may be due to the low signal to noise ratio as measured by the sensors.

The identified model was then used to design a number of resonant controllers, which were subsequently implemented on the system. This is detailed in the following subsection.

\section{E. Controller Design}

Two resonant controllers were proposed in Section VI. Of the two controllers, (17) rolls off faster at higher frequencies, and is therefore chosen as the candidate controller for the above structure.

To obtain an effective controller, appropriate values for $d_{i}$, $\tilde{\omega}_{i}$ and $\beta_{i}$ for each mode need to be selected. This can be achieved in a number of ways. For example, by minimizing the $H_{2}$ or $H_{\infty}$ norm of the closed loop transfer function from disturbance voltage to tip displacement $\left(T_{Y_{\text {tip }} w}\right)$. However, more than simply minimizing a specific measure, the ultimate purpose of the controller is to add extra damping to the system by shifting the closed loop poles of the plant deeper into the left half of the complex plane. This will ensure that no matter where a disturbance is entering the system the structural vibration is minimized within the controlled bandwidth.

A number of performance measures were used to determine appropriate parameters for the controller. Only two of these methods are reported in this paper. The first controller was obtained by minimizing the $H_{2}$ norm of $T_{Y_{\text {tip }} w}$, and the second by placing the poles of the closed-loop system as far to the left of the $j \omega$ axis as possible.

The optimizations were carried out using the simplex search algorithm in MATLAB. Before performing the optimization, the values of $\beta$ were fixed such that condition (15) was satisfied.

The values of $\beta$ were chosen manually taking into account the authority of the controller over each specific mode, through either actuator. In practice this means the need to select relatively large absolute numbers for the first mode associated with the first actuator, and for the second and third modes associated with the second actuator. The numbers must be chosen such that (15) is satisfied. The values used in the experiments are

$$
\beta=\left[\begin{array}{ccc}
0.9722 & -0.2308 & 0.0399 \\
0.2046 & 0.7541 & -0.6241
\end{array}\right]
$$


The first and second row correspond to the first and second collocated arrangements respectively. The column entries correspond to their respective modes as well. ${ }^{6}$

Since the $\beta$ values are fixed, only the $\tilde{\omega}$ and $d$ values needed to be determined using the optimization routine.

Minimizing the $\mathrm{H}_{2}$ norm of the closed loop system yields a resonant controller that adds damping to the structure, in a roundabout way. The pole placement technique, however, is a more straightforward approach since the physical location of the closed-loop poles is intrinsically included in the cost function. In this approach, the absolute distance between a prespecified pole location and the actual closed-loop poles of the system is minimized.

A Bode plot of the multivariable $\mathrm{H}_{2}$ controller is shown in Fig. 16. As can be noted, the diagonal entries correspond to the collocated transfer functions. Also due to (15), the cross diagonal transfer functions are identical.

\section{EXPERIMENTAL RESULTS}

The experiments were performed in the Laboratory for Dynamics and Control of Smart Structures at the University of Newcastle, Australia, and were carried out on a cantilever Euler Beam with identical collocated PIC 151 piezoelectric patches, with dimensions shown in Fig. 13. The disturbance voltage was applied to a secondary patch located at the center of the beam and the two collocated actuator-sensor pairs were used for feedback control purposes only. A Polytec laser scanning vibrometer (PSV-300) was used to measure the velocity at the tip of the beam.

In this experiment, the frequency responses were obtained by applying a sinusoidal voltage signal of varying frequency to the "disturbance" piezoelectric patch and measuring the corresponding output tip displacement of the beam $\left(Y_{\text {tip }}\right)$, with and without the controller being switched on, using a similar procedure as described in Section VII-D. The Polytec software was again used to determine the corresponding frequency responses.

A schematic of the experimental testbed is shown in Fig. 14. To carry out the experiments, the controller was downloaded from Simulink onto a dSPACE DS-1103 DSP board with the sampling rate set at $20 \mathrm{KHz}$. Low-pass antialiasing, and reconstruction filters were added to the system. These were secondorder Butterworth filters with a cut-off frequency of $3 \mathrm{KHz}$. The measured voltage for each piezoelectric sensor was passed through a high-impedance buffer. This ensured the sensor signals were not distorted at low frequencies.

The measured and simulated open- and closed-loop Bode magnitude plots for the $\mathrm{H}_{2}$ optimized resonant controller are given in Fig. 17(a) and (b), respectively. The same plots for the pole placement optimization method are shown in Fig. 19. On average these controllers reduced the resonant peak of the input disturbance $(w)$ to output displacement $\left(Y_{\text {tip }}\right)$ transfer function by $13 \mathrm{~dB}$ for each mode.

Time domain displacement step responses for the $H_{2}$ and pole placement optimized resonant controllers are displayed in

\footnotetext{
${ }^{6}$ The choice of $\beta$ as proposed here is rather ad hoc. It is possible, however, to choose an appropriate value for $\beta$ by making an initial guess and then incorporating that into the overall constrained optimization problem.
}

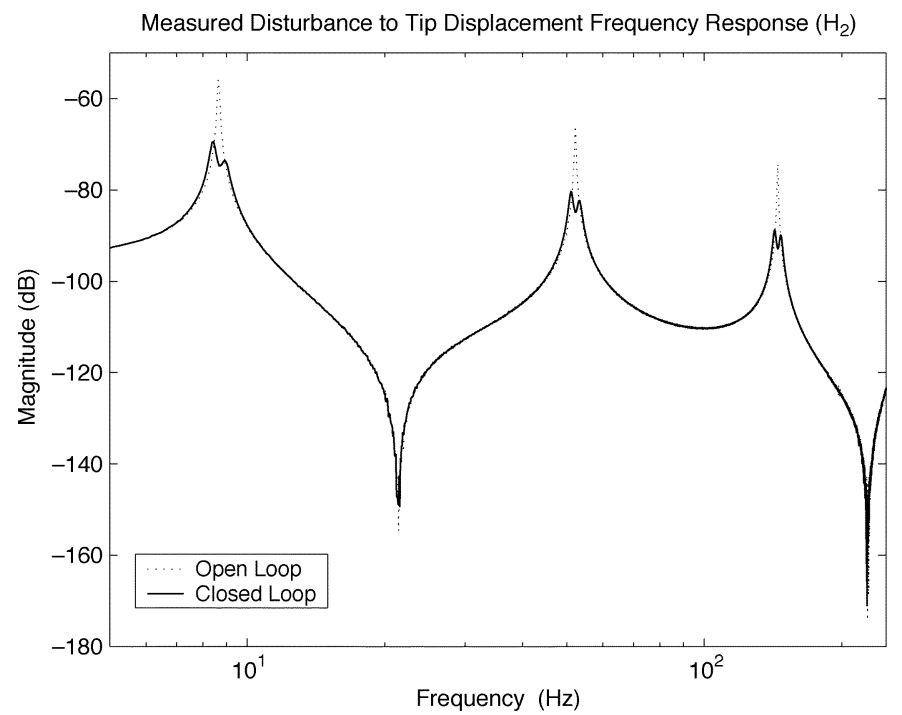

(a)

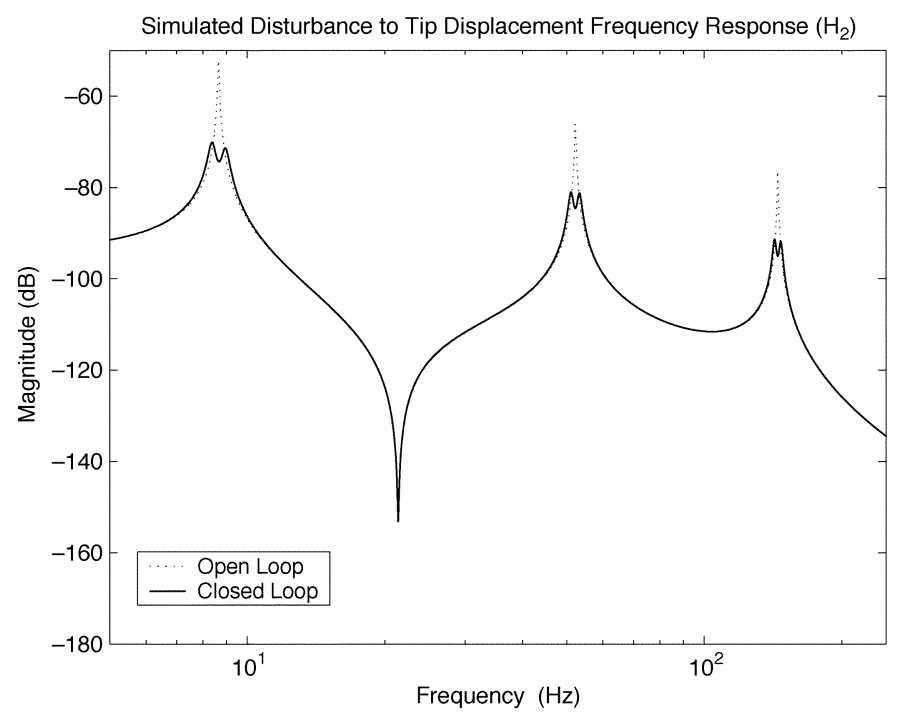

(b)

Fig. 17. Open- and closed-loop frequency response for input disturbance $w$ to output tip displacement $Y_{\text {tip }}$ using the $H_{2}$ norm optimization. (a) Measured data. (b) Simulation results.

Figs. 18 and 20, respectively. These plots were obtained by applying a low-pass filtered $(250 \mathrm{~Hz})$ step signal to the disturbance patch and measuring the velocity at the tip of the beam. The velocity was then integrated off line to obtain the corresponding displacement. The open loop step response is also included in these plots as a comparison. Both control schemes performed well and reduced the settling time of the beam by a factor of 5 .

In this experiment, the multivariable pole placement charge controller $k_{q}$ was used to determine the equivalent voltage controller $K_{v}$ via the (12). Then all unused piezoelectric transducers were short circuited, and only the first controller channel was left active. A comparison of the open-loop and closed-loop responses of the system in Fig. 22 as measured by the laser vibrometer with 10 and 200 volt swept-sine signals applied to the disturbance patch, shows a performance degradation of over $1 \mathrm{~dB}$ at resonance, which is mainly due to the existence of hysteresis when the piezoelectric actuators are driven by voltage amplifiers at high drives. 

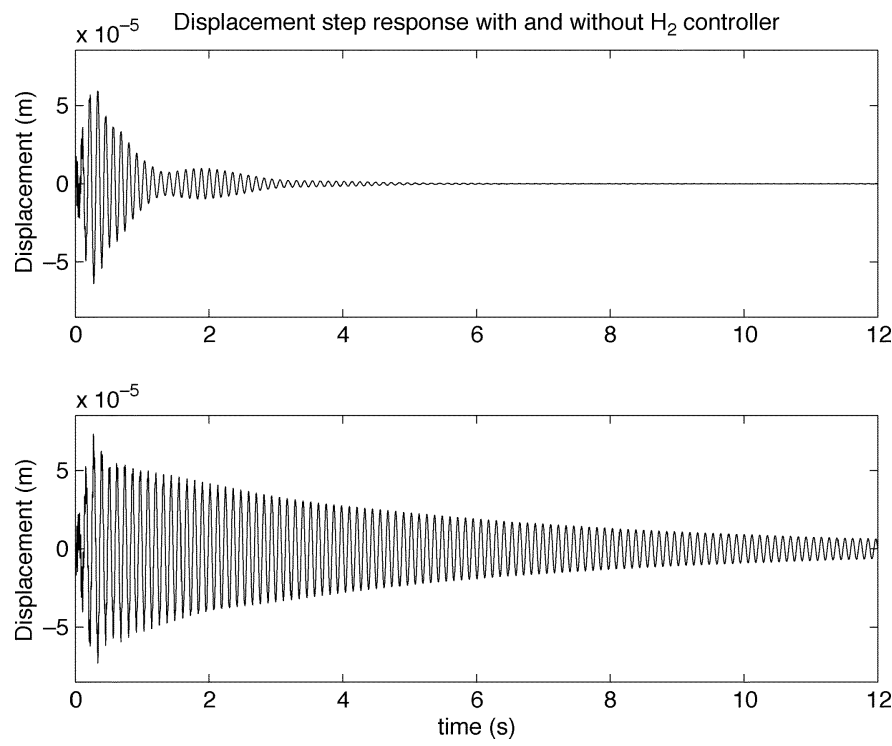

Fig. 18. Displacement step response at the tip of the beam with and without controller.

Finally, to illustrate the drawbacks of using voltage controlled piezoelectric actuators a number of experiments were performed using the first collocated piezoelectric actuator/sensor pair. Fig. 21 presents the Bode plot of the first mode of the system when the piezoelectric actuator closest to the clamped end of the beam is driven by a voltage amplifier and the voltage induced in the collocated sensor is taken as the output. The two plots, one obtained using a swept-sine signal with an amplitude of 2 volts and another with an amplitude of 130 volts, clearly illustrate the effect of hysteresis. The hysteresis manifests itself in the form of a phase lag of about $6.8^{\circ}$, and a shift in amplitude and resonance frequency. ${ }^{7}$

Closed-loop experiments were also conducted using a multivariable voltage controller $K_{v}$ which is (from a closed-loop point of view) equivalent to the multivariable pole placement charge driven controller $K_{q}$ via (12). All unused piezoelectric transducers were short circuited, and only the first controller channel was left active. A comparison of the open-loop and closed-loop responses of the system in Fig. 22 as measured by the laser vibrometer with 15 and 150 volt swept-sine signals applied to the disturbance patch, shows a performance degradation of over $1 \mathrm{~dB}$ at resonance, which is mainly due to the existence of hysteresis when the piezoelectric actuators are driven by voltage amplifiers at high drives. The phase for the high voltage scenario also has a uniform lag of $4.5^{\circ}$ over the measured bandwidth. Unlike charge, these results clearly demonstrate that the use of voltage to drive piezoelectric actuators can result in performance degradation, due to the existence of hysteresis at high drives.

\section{OBSERVATIONS AND DISCUSSION}

The two optimization schemes produced acceptable results. Indeed the closed-loop response for these controllers demon-

\footnotetext{
${ }^{7}$ This is somewhat expected and is in line with observations made in [11] and [8]. The first harmonic approximation of a hysteresis curve is an ellipse, which amounts to a phase shift.
}

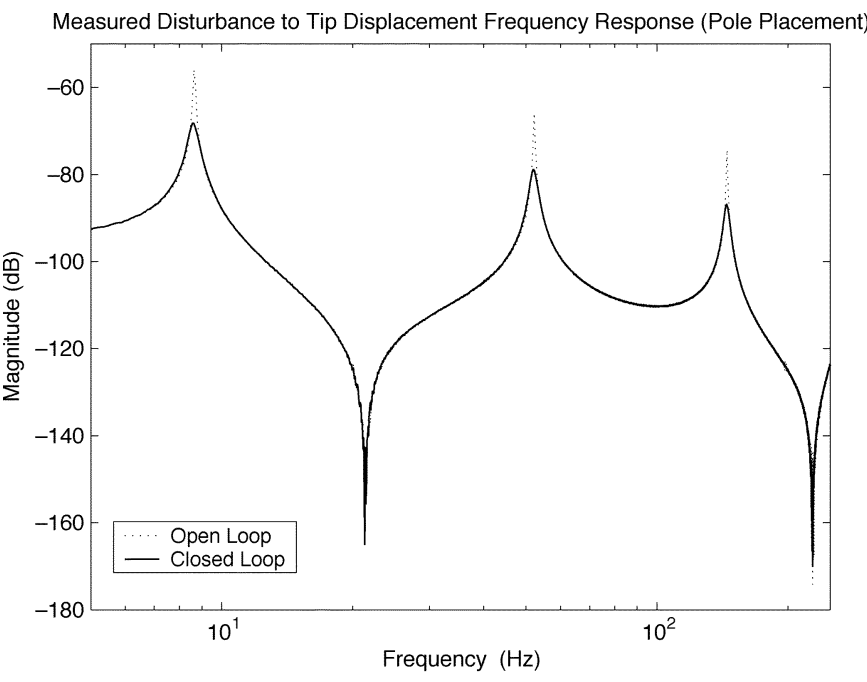

(a)

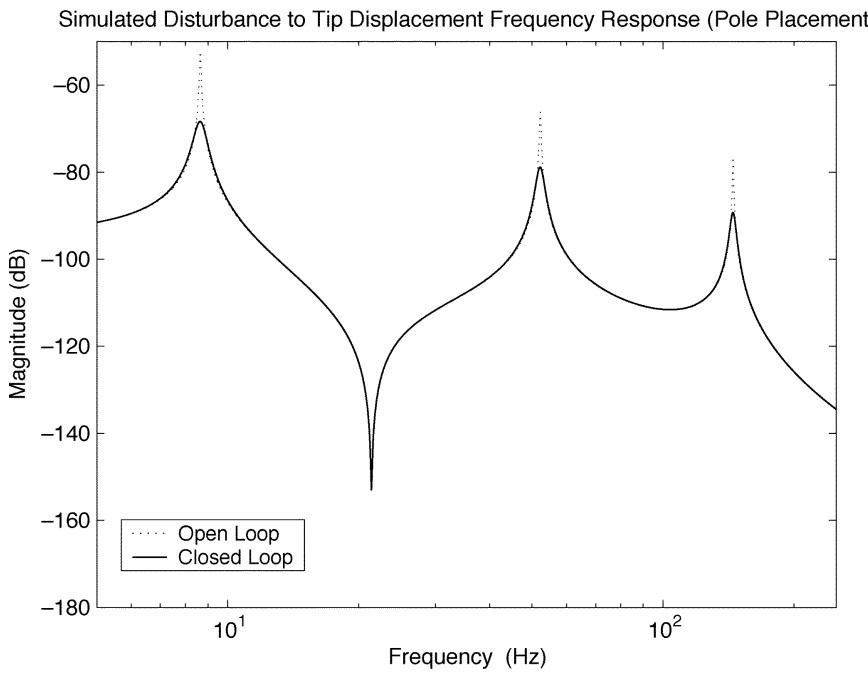

(b)

Fig. 19. Open- and closed-loop frequency response for input disturbance $w$ to output tip displacement $Y_{\text {tip }}$ using the pole placement optimization. (a) Measured data. (b) Simulation results.

strate significant damping. The damping ratios $\left(\zeta_{i}\right)$ associated with each mode of the closed-loop system were determined for each controller. The results are given in Table I. Notice that in each case only the damping ratio associated with the pole closest to the $j \omega$ axis is shown.

It can be observed that the pole placement optimization method provides the most damping for each mode. This can be verified by looking at the closed-loop pole locations for each optimization scheme (Fig. 23) since the closed-loop poles associated with the pole placement method are located furthest to the left from the imaginary axis.

\section{Conclusion}

This paper has demonstrated that charge driven piezoelectric resonant controllers can successfully be used in structural vibration control applications to reject unwanted disturbances entering the plant. These fixed-structure controllers offer interesting properties such as stability robustness in presence of out-of-bandwidth dynamics, and good damping performance. 

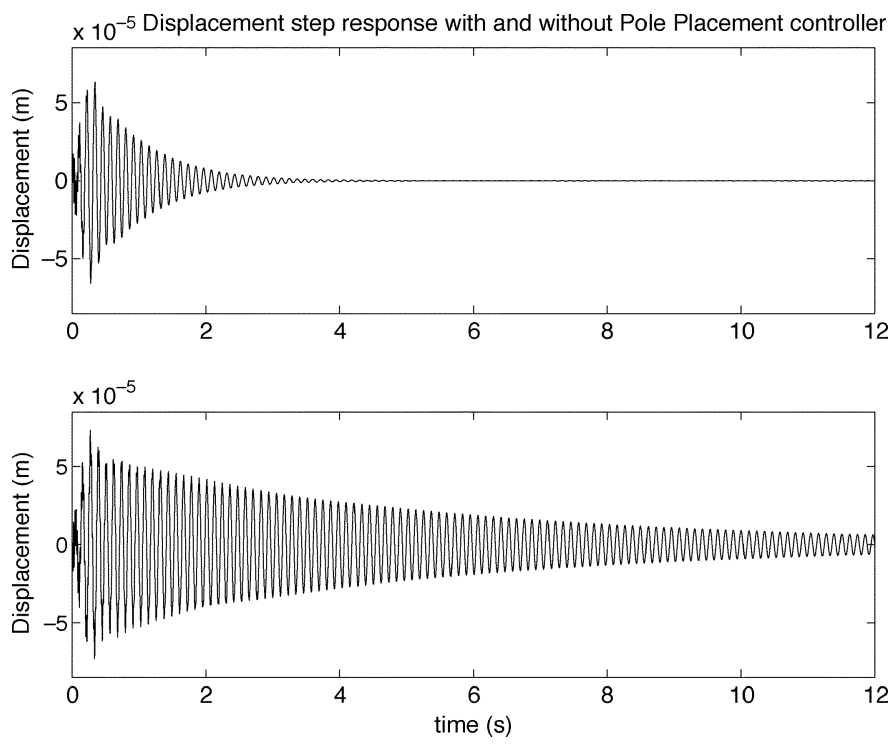

Fig. 20. Displacement step response at the tip of the beam with and without controller.

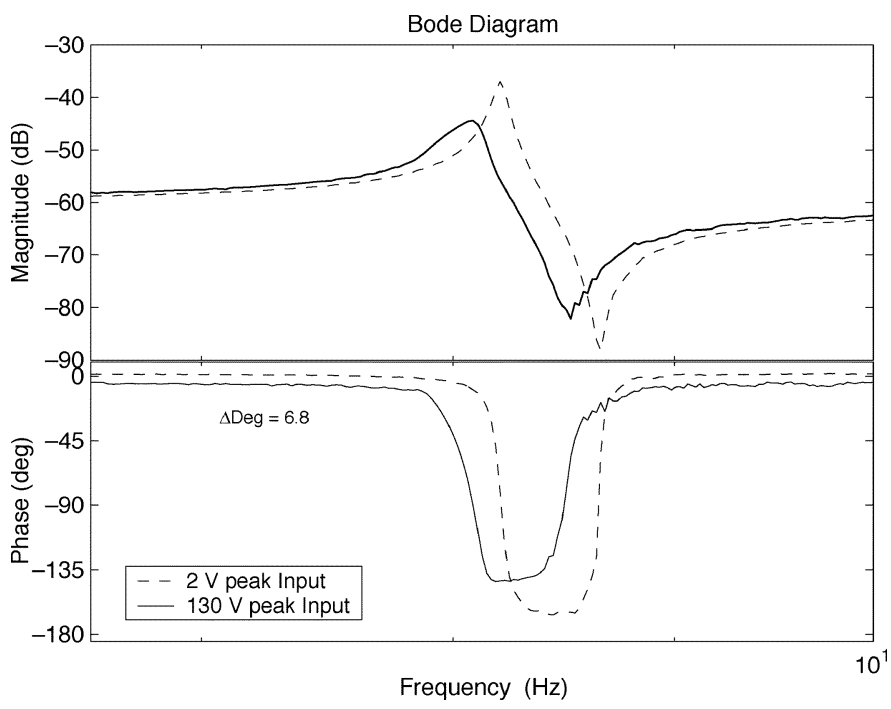

Fig. 21. Bode plot of the first mode of $G_{v v}$, at low and high drives.

However, there are difficulties associated with their use when voltage is used to drive the piezoelectric actuators. The hysteretic nature of the actuator, as well as the slow roll off of the system, complicate the operation of the voltage driven feedback controller. This paper explained how the structure of resonant controllers can be modified when piezoelectric actuators are driven by charge amplifiers. In this case the underlying system is, to a large extent, free of hysteresis. Furthermore, the modified resonant controllers roll off quickly at higher frequencies, which compensates for the natural slow roll off of the system.

\section{APPENDIX}

This appendix contains proof of stability of the closed loop system under the two resonant controllers proposed in Section III.

Theorem 1: Feedback connection of (1) and (5) is stable.
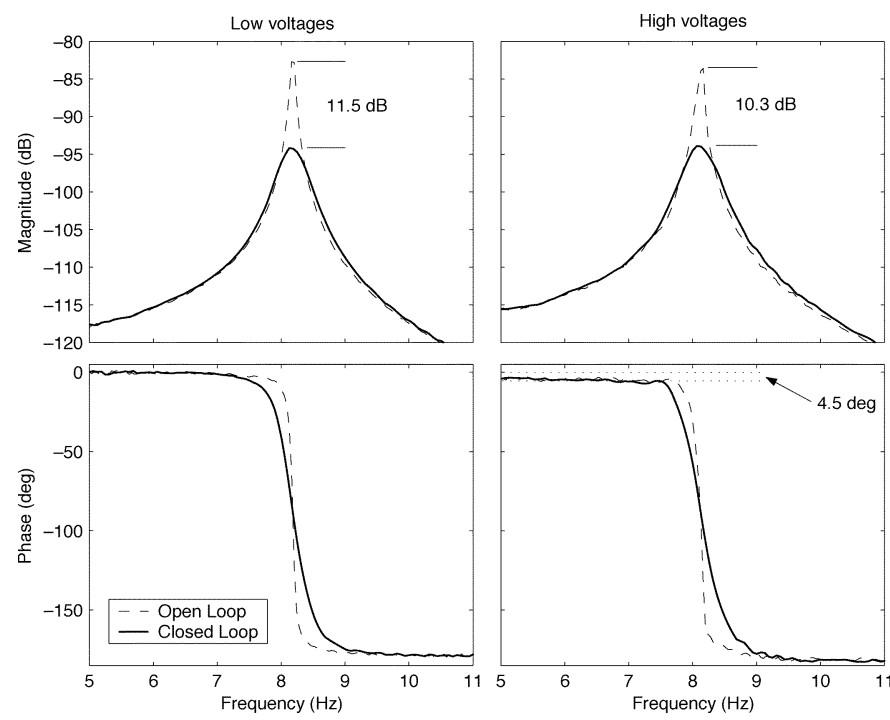

Fig. 22. Open-loop and closed-loop performance with high and low level disturbances.

TABLE I

DAMPING RATIOS

\begin{tabular}{l|c|c|c}
\hline & \multicolumn{3}{|c}{ Damping ratios $\left(10^{-3}\right)$} \\
\hline Modes & $1^{\text {st }}$ & $2^{\text {nd }}$ & $3^{\text {rd }}$ \\
\hline Open Loop & 3.29 & 3.26 & 2.35 \\
\hline $\mathrm{H}_{2}$ norm & 18.9 & 12.5 & 9.15 \\
\hline Pole placement & 39.8 & 26.2 & 19.2 \\
\hline
\end{tabular}

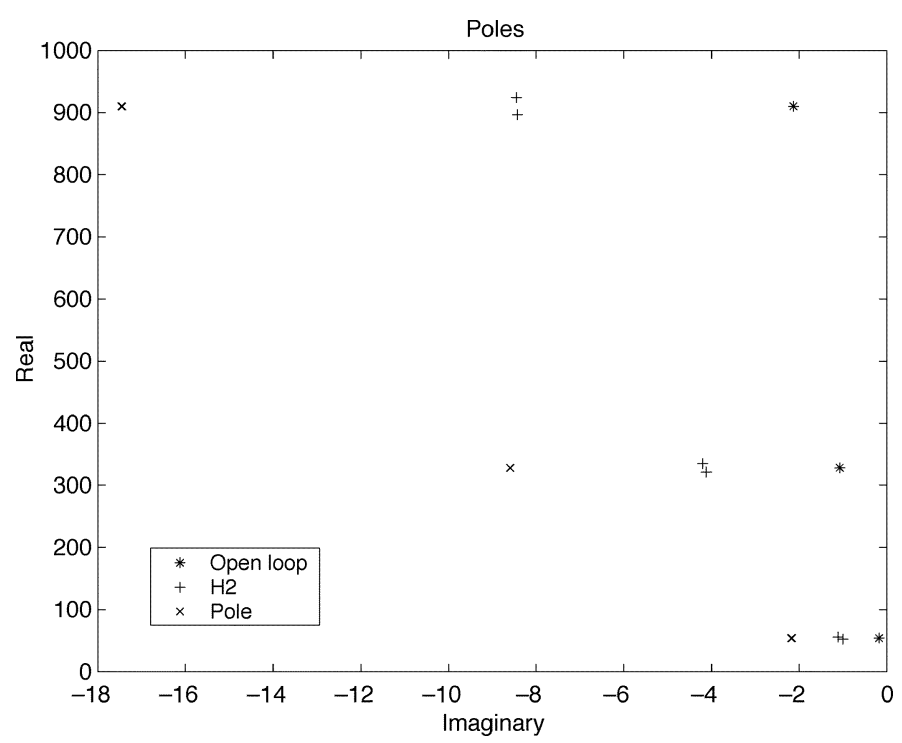

Fig. 23. Comparison of the closed-loop pole locations for the different resonant controllers (second quadrant shown only).

Proof: Stability of (1) and (5) is equivalent to the stability of $^{8}$ (see also [43])

$$
\tilde{G}_{v v}(s)=\sum_{i=1}^{M} \frac{\psi_{i} \psi_{i}^{\prime} s}{s^{2}+2 \zeta_{i} \omega_{i} s+\omega_{i}^{2}}
$$

${ }^{8}$ Here, and in the next theorem we are assuming that $\zeta_{i}>0, d_{i}>0$ for all $i$. 
and

$$
\tilde{K}_{v}^{\beta}=\sum_{i=1}^{\tilde{N}} \frac{\beta_{i} \beta_{i}^{\prime}\left(s+2 d_{i} \tilde{\omega}_{i}\right)}{s^{2}+2 d_{i} \tilde{\omega}_{i} s+\tilde{\omega}_{i}^{2}} .
$$

Now, it can be proved that $\tilde{G}_{v v}(s)$ is a positive-real (PR) transfer function matrix, while $\tilde{K}_{v}^{\beta}$ is a strictly positive-real (SPR) transfer function matrix. Negative feedback connection of a PR and a SPR system is known to be stable [44]-[46].

Closed loop stability of (1) under (4) can be established by constructing a Lyapunov function.

Theorem 2: Feedback connection of (1) and (4) is exponentially stable. ${ }^{9}$

Proof: Feedback connection of (1) and (4) is stable if and only if the closed loop system depicted in Fig. 24 is stable, where

$$
\tilde{G}_{v v}(s)=\sum_{i=1}^{M} \frac{\psi_{i} \psi_{i}^{\prime} s}{s^{2}+2 \zeta_{i} \omega_{i} s+\omega_{i}^{2}}
$$

and

$$
\tilde{K}_{v}^{\alpha}=\sum_{i=1}^{\tilde{N}} \frac{\alpha_{i} \alpha_{i}^{\prime} s}{s^{2}+2 d_{i} \tilde{\omega}_{i} s+\tilde{\omega}_{i}^{2}} .
$$

Now, $\tilde{G}_{v v}(s)$ can be represented by the second-order state-space equations

$$
\begin{aligned}
\ddot{x}(t)+2 Z \Omega \dot{x}(t)+\Omega^{2} x(t) & =\Psi^{\prime} u(t) \\
y(t) & =\Psi \dot{x}(t)
\end{aligned}
$$

where

$$
\begin{aligned}
& Z=\left[\begin{array}{llll}
\zeta_{1} & & & \\
& \zeta_{2} & & \\
& & \ddots & \\
& & & \zeta_{N}
\end{array}\right] \\
& \Omega=\left[\begin{array}{llll}
\omega_{1} & & & \\
& \omega_{2} & & \\
& & \ddots & \\
& & & \omega_{N}
\end{array}\right] \\
& \Psi=\left[\begin{array}{llll}
\psi_{1} & \psi_{2} & \ldots & \psi_{N}
\end{array}\right] .
\end{aligned}
$$

Furthermore, $\tilde{K}_{v}^{\alpha}(s)$ can be written as

$$
\begin{aligned}
\ddot{\tilde{x}}(t)+2 D \tilde{\Omega} \dot{x}(t)+\tilde{\Omega}^{2} x(t) & =\Gamma^{\prime} y(t) \\
u(t) & =-\Gamma \dot{\tilde{x}}(t)
\end{aligned}
$$

where

$$
\begin{aligned}
& D=\left[\begin{array}{llll}
d_{1} & & & \\
& d_{2} & & \\
& & \ddots & \\
& & & d_{N}
\end{array}\right] \\
& \tilde{\Omega}=\left[\begin{array}{llll}
\tilde{\omega}_{1} & & & \\
& \tilde{\omega}_{2} & & \\
& & \ddots & \\
& & & \tilde{\omega}_{N}
\end{array}\right] \\
& \Gamma=\left[\begin{array}{llll}
\alpha_{1} & \alpha_{2} & \ldots & \alpha_{N}
\end{array}\right] .
\end{aligned}
$$

\footnotetext{
${ }^{9}$ We point out that a similar proof is given in [47]. The proof included here is for the sake of completeness.
}

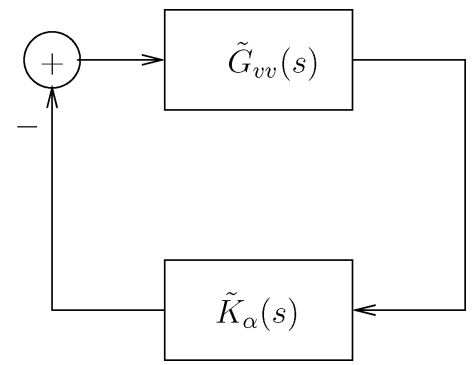

Fig. 24. Feedback structure associated with charge-driven piezoelectric actuator/sensor pairs.

Combining (29) and (30), the closed-loop system dynamics can be obtained

$\left[\begin{array}{c}\ddot{x}(t) \\ \ddot{\tilde{x}}(t)\end{array}\right]+\left[\begin{array}{cc}2 Z \Omega & -\Psi^{\prime} \Gamma \\ -\Gamma^{\prime} \Psi & 2 D \tilde{\Omega}\end{array}\right]\left[\begin{array}{c}\dot{x}(t) \\ \tilde{\tilde{x}}(t)\end{array}\right]+\left[\begin{array}{cc}\Omega^{2} & 0 \\ 0 & \tilde{\Omega}^{2}\end{array}\right]\left[\begin{array}{l}x(t) \\ \tilde{x}(t)\end{array}\right]=\left[\begin{array}{l}0 \\ 0\end{array}\right]$.

Now, a Lyapunov function, $V(x(t), \tilde{x}(t))$ can be defined as

$V(x(t), \tilde{x}(t))=\left[\begin{array}{l}\dot{x}(t) \\ \tilde{x}(t)\end{array}\right]^{\prime}\left[\begin{array}{l}\dot{x}(t) \\ \tilde{x}(t)\end{array}\right]+\left[\begin{array}{l}x(t) \\ \tilde{x}(t)\end{array}\right]^{\prime}\left[\begin{array}{cc}\Omega^{2} & 0 \\ 0 & \tilde{\Omega}^{2}\end{array}\right]\left[\begin{array}{l}x(t) \\ \tilde{x}(t)\end{array}\right]$.

Clearly, $V(x(t), \tilde{x}(t))>0$ for all nontrivial $x, \dot{x}, \tilde{x}$, and $\dot{\tilde{x}}$. Differentiating $V$ with respect to time yields

$$
\dot{V}(x(t), \tilde{x}(t))=\left[\begin{array}{l}
\dot{x}(t) \\
\dot{\tilde{x}}(t)
\end{array}\right]^{\prime}\left[\begin{array}{cc}
-2 Z \Omega & -\Psi^{\prime} \Gamma \\
-\Gamma^{\prime} \Psi & -2 D \tilde{\Omega}
\end{array}\right]\left[\begin{array}{l}
\dot{x}(t) \\
\dot{\tilde{x}}(t)
\end{array}\right]
$$

which implies

$$
\dot{V}(x(t), \tilde{x}(t))<0
$$

for nontrivial values of $\dot{x}$ and $\dot{\tilde{x}}$. Therefore, the closed-loop system is asymptotically stable.

The closed-loop charge-driven system under controllers (16) and (17), is identical to the voltage-driven system under controllers (4) and (5). Given that closed-loop stability of the latter has been established, closed-loop stability of the former system follows immediately.

\section{REFERENCES}

[1] T. Ikeda, Fundamentals of Piezoelectricity. Oxford, U.K.: Oxford Univ. Press, 1996.

[2] P. Ge and M. Jouaneh, "Modeling hysteresis in piezoceramic actuators," Precision Eng., vol. 17, pp. 211-221, 1995.

[3] M. Goldfarb and N. Celanovic, "A lumped parameter electromechanical model for describing the nonlinear behavior of piezoelectric actuators," ASME J. Dyna. Syst.,Measure., Control, vol. 119, pp. 478-485, Sep. 1997.

[4] C. Newcomb and I. Flinn, "Improving the linearity of piezoelectric actuators using charge feedback," Electron. Lett., vol. 18, no. 11, pp. 442-444, May 1982.

[5] J. A. Main and E. Garcia, "Piezoelectric stack actuators and control system design: strategies and pitfalls," AIAA J. Guid., Control Dyna., vol. 20, no. 3, pp. 479-485, 1997.

[6] K. Furutani, M. Urushibata, N. Mohri, and N. May, "Improvement of control method for piezoelectric actuator by combining charge feedback with inverse transfer function compensation," in Proc. IEEE Int. Conf. Robotics and Automation, Leuven, Belgium, 1998, pp. 1504-1509.

[7] I. Mayergoyz, Mathematical Models of Hysteresis. New York: Springer-Verlag, 1991.

[8] J. M. Cruz-Hernandez and V. Hayward, "Phase control approach to hysteresis reduction," IEEE Trans. Control Syst. Technol., vol. 9, no. 1, pp. 17-26, Jan. 2001. 
[9] R. C. Smith, Z. Ounaies, and R. Wieman, "A Model for rate-dependent hysteresis in piezoceramic materials operating at low frequencies,", NASA/CR-2001-211 062 ICASE Rep. 2001-26, 2001.

[10] B. M. Chen, T. H. Lee, H. Chang-Chieh, Y. Guo, and S. Weerasooriya, "An $H_{\infty}$ almost disturbance decoupling robust controller design for a piezoelectric bimorph actuator with hysteresis," IEEE Trans. Control Syst. Technol., vol. 7, no. 2, pp. 160-174, Feb. 1999.

[11] J. A. Main and E. Garcia, "Design impact of piezoelectric actuator nonlinearities," AIAA J. Guid., Control, Dyna., vol. 20, no. 2, pp. 327-332, 1997.

[12] J. A. Main, E. Garcia, and D. V. Newton, "Precision position control of piezoelectric actuators using charge feedback," AIAA J. Guid., Control, Dyna., vol. 18, no. 5, pp. 1068-1073, 1995.

[13] A. J. Fleming and S. O. R. Moheimani, "Precision current and charge amplifiers for driving highly capacitive piezoelectric loads," Electron. Lett., vol. 39, no. 3, pp. 282-284, Feb. 2003.

[14] D. Halim and S. O. R. Moheimani, "Spatial resonant control of flexible structures-application to a piezoelectric laminate beam," IEEE Trans. Control Syst. Technol., vol. 9, no. 1, pp. 37-53, Jan. 2001.

[15] H. R. Pota, S. O. R. Moheimani, and M. Smith, "Resonant controllers for smart structures," Smart Mater. Struct., vol. 11, no. 1, pp. 1-8, 2002.

[16] E. F. Crawley and J. de Luis, "Use of piezoelectric actuators as elements of intelligent structures," AIAA J., pp. 1373-1385, Oct. 1987.

[17] C. K. Lee, "Piezoelectric laminates for torsional and bending modal control: Theory and experiment," Ph.D. dissertation, Cornell Univ., Ithaca, NY, 1987

[18] A. Baz and S. Poh, "Performance of an active control system with piezoelectric actuators," J. Sound Vibrat., vol. 126, no. 2, pp. 327-343, 1988.

[19] H. H. Cudney, "Distributed structural control using multilayered piezoelectric actuators," Ph.D. dissertation, SUNY, Buffalo, NY, 1989.

[20] H. S. Tzou, "Integrated distributed sensing and active vibration suppression of flexible manipulators using distributed piezoelectrics," J. Robot. Syst., vol. 6, no. 6, pp. 745-767, 1989.

[21] N. W. Hagood, W. H. Chung, and A. von Flotow, "Modeling of piezoelectric actuator dynamics for active structural control," J. Intell. Mater. Syst. Struct., vol. 1, no. 3, pp. 327-354, 1990.

[22] I. R. Petersen and H. R. Pota, "Minimax LQG optimal control of a flexible beam," Control Eng. Practice, vol. 11, no. 11, pp. 1273-1287, Nov. 2003.

[23] F. Casella, A. Locatelli, and N. Schiavoni, "Modeling and control for vibration suppression in a large flexible structure with jet thrusters and piezoactuators," IEEE Trans. Control Syst. Technol., vol. 10, no. 4, pp. 589-599, Apr. 2002.

[24] S. O. R. Moheimani, D. Halim, and A. J. Fleming, Spatial Control of Vibration: Theory and Experiments. Singapore: World Scientific, 2003.

[25] C. W. de Silva, Vibration Fundamentals and Practice. Boca Raton, FL: CRC Press, 1999

[26] H. R. Pota and T. E. Alberts, "Multivariable transfer functions for a slewing piezoelectric laminate beam," ASME J. Dyna. Syst., Measure., Control, vol. 117, pp. 353-359, Sep. 1995.

[27] T. E. Alberts and J. A. Colvin, "Observations on the nature of transfer functions for control of piezoelectric laminates," J. Intell. Mater. Syst. Struct., vol. 8, no. 5, pp. 605-611, 1991.

[28] P. C. Hughes, "Space structure vibration modes: how many exist? which ones are important?," IEEE Control Syst. Mag., vol. 8, no. 1, pp. 22-28, Feb. 1987.

[29] J. Juang and M. Q. Phan, Identification and Control of Mechanical Systems. Cambridge, U.K.: Cambridge Univ. Press, 2001.

[30] R. L. Clark, "Accounting for out-of-bandwidth modes in the assumed modes approach: implications on colocated output feedback control," Trans. ASME J. Dyna. Syst., Measure., Control, vol. 119, pp. 390-395, 1997.

[31] S. O. R. Moheimani, "Minimizing the effect of out of bandwidth modes in truncated structure models," Trans. ASME J. Dyna. Syst., Measure., Control, vol. 122, no. 1, pp. 237-239, Mar. 2000.

[32] G. D. Martin, "On the control of flexible mechanical systems," Ph.D. dissertation, Stanford Univ., Stanford, CA, 1978.

[33] A. Preumont, Vibration Control of Active Structures. Norwell, MA: Kluwer, 1997.

[34] J. L. Fanson and T. K. Caughey, "Positive position feedback control for large space strcture," in Proc. 28th AIAA/ASME/ASC/AHS Structures Structural Dynamics and Materials Conf., Monterey, California, 1987, pp. 588-598.

[35] M. J. Balas, "Feedback control of flexible systems," IEEE Trans. Autom. Control, vol. AC-23, no. 4, Jul. 1978.
[36] S. O. R. Moheimani, A. J. Fleming, and S. Behrens, "Dynamics, stability and control of multivariable piezoelectric shunts," IEEE/ASME Trans. Mechatron., vol. 9, no. 1, pp. 87-99, Mar. 2004.

[37] — "On the feedback structure of wide-band piezoelectric shunt damping systems," Smart Mater. Struct., vol. 12, no. 1, pp. 49-56, Feb. 2003.

[38] R. L. Clark, W. R. Saunders, and G. P. Gibbs, Adaptive Structures: Dynamics \& Control. New York: Wiley, 1998.

[39] D. Croft, G. Shed, and S. Devasia, "Creep, hysteresis, and vibration compensation for piezoactuators: atomic force microscopy application,' ASME J. Dyna. Syst. Control, vol. 123, no. 1, pp. 35-43, Mar. 2001.

[40] D. Croft and S. Devasia, "Vibration compensation for high speed scanning tunneling microscopy," Rev. Sci.Instrum., vol. 70, no. 12, pp. 4600-4605, Dec. 1999.

[41] R. Comstock, "Charge control of piezoelectric actuators to reduce hysteresis effect," U.S. Patent 4.263 527, 1981.

[42] S. O. R. Moheimani, "Experimental verification of the corrected transfer function of a piezoelectric laminate beam," IEEE Trans. Control Syst. Technol., vol. 8, no. 4, pp. 660-666, Jul. 2000.

[43] R. J. Benhabib, R. P. Iwens, and R. L. Jackson, "Stability of larges space structures uing positivity concepts," J. Guid. Control, vol. 5, pp. 487-494, Sep.-Oct. 1981.

[44] V. M. Popov, Hyperstability of Control Systems. New York: SpringerVerlag, 1973.

[45] C. H. Huang, P. A. Ioannou, J. Maroulas, and M. G. Safanov, "Design of strictly positive real systems using constant output," IEEE Trans. Autom. Control, vol. 44, no. 3, pp. 569-572, Mar. 1999.

[46] B. D. O. Anderson, "A simplified viewpoint on hyperstability," IEEE Trans. Autom. Control, vol. AC-13, no. 3, pp. 292-294, Jun. 1968.

[47] E. Sim and S. W. Lee, "Active vibration control of flexible structures with acceleration or combined feedback," AIAA J. Guid., Control, Dyna., vol. 16, no. 2, pp. 413-415, 1993.

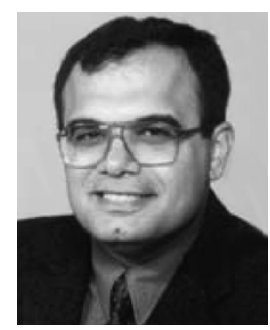

S. O. Reza Moheimani (S'93-M'97-SM'00) received the B.Sc. degree from Shiraz University, Shiraz, Iran, in 1990, and the M.Eng.Sc. and Ph.D. degrees from the University of New South Wales, Australia, in 1993 and 1996, respectively, all in electrical and electronics engineering.

In 1996, he was a Postdoctoral Research Fellow at the School of Electrical and Electronics Engineering, Australian Defence Force Academy, Canberra. In 1997, he joined the University of Newcastle, Australia, where he is currently an Associate Professor in the School of Electrical Engineering and Computer Science. He has over 100 journal and conference publications, is a coauthor of the research monograph Spatial Control of Vibration: Theory and Experiments (Singapore: World Scientific, 2003) and the editor of the volume Perspectives in Robust Control (New York: Springer-Verlag, 2001). His research interests include smart structures, mechatronic systems, control theory, and signal processing.

Dr. Moheimani is an Associate Editor of the IEEE TRANSACTIONS ON Control Systems TeChNOLOGY and the International Journal of Control, Automation, and Systems, and a past Associate Editor of Control Engineering Practice. He has served on the Editorial Boards of several international conferences, and was the Chairman of International Program Committee for the 3rd IFAC Symposium on Mechatronic Systems, held in Sydney, Australia, in September 2004. He is a Member of the ARC Centre for Complex Dynamic Systems and Control, where he directs the Centre's research in the area of mechatronics.

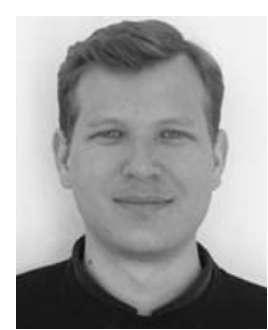

Benjamin J. G. Vautier was born in 1978. He received the B.Sc. degree in mechanical engineering from the University of Western Australia in 2002, and the M.Eng. degree in electrical engineering from the University of Newcastle, Australia, in 2004.

His research interests include understanding and compensating for hysteresis in piezoelectric actuators when used in structural vibration control applications. 